Check for updates

Cite this: Phys. Chem. Chem. Phys., $2018,20,16077$

Received 6th April 2018,

Accepted 14th May 2018

DOI: $10.1039 / c 8 c p 02188 k$

rsc.li/pccp

\section{Chemical and substitutional doping, and anti-site and vacancy formation in monolayer AIN and GaN $\dagger$}

\author{
Yelda Kadioglu, ${ }^{a b}$ Fatih Ersan, ${ }^{a b}$ Deniz Kecik, ${ }^{\text {bc }}$ Olcay Üzengi Aktürk, de \\ Ethem Aktürk (D) *e and Salim Ciraci*b
}

\begin{abstract}
We investigated the effects of chemical/substitutional doping, hydrogenation, and anti-site and vacancy defects on the atomic, optoelectronic and magnetic properties of AIN and GaN monolayers. Upon doping of selected atoms, AIN and GaN monolayers can acquire magnetic properties, and their fundamental band gaps are modified by the localized gap states. Spin-polarized gap states broaden into bands at patterned coverage of adatoms, whereby half-metallic or magnetic semiconducting properties can be attained. Specific adatoms adsorbed to $\mathrm{Ga}$ atoms break the nearest vertical $\mathrm{Ga}-\mathrm{N}$ bonds in the GaN bilayer in the heackelite structure and result in changes in the electronic and atomic structure. While adjacent and distant pairs of anion + cation vacancies induce spin polarization with filled and empty gap states, anti-site defects remain nonmagnetic; but both defects induce dramatic changes in the band gap. Fully hydrogenated monolayers are stable only for specific buckled geometries, where one geometry can also lead to an indirect to direct band gap transition. Also, optical activity shifts to the ultra-violet region upon hydrogenation of the monolayers. While $\mathrm{H}_{2}$ and $\mathrm{O}_{2}$ molecules are readily physisorbed on the surfaces of the monolayers with weak van der Waals attraction, they can be dissociated into constituent atoms at the vacancy site of the cation. Our study performed within density functional theory shows that the electronic, magnetic and optical properties of AIN and GaN monolayers can be tuned by doping and point defect formation in order to acquire diverse functionalities.
\end{abstract}

\section{Introduction}

As various two-dimensional (2D) monolayer structures, like h-BN and $\mathrm{h}-\mathrm{MoS}_{2}$, have been exploited as a competitor of graphene, the possibility that Si and group III-V compounds, such as GaAs, $\mathrm{GaN}$ and AlN can form graphene-like structures was predicted already in 2005 by $a b$ initio calculations. ${ }^{1}$ Later, the stability of monolayer honeycomb structures of group IV elements, Si, Ge, $\mathrm{SiC}$ and several group III-V compounds including GaN and AlN

\footnotetext{
${ }^{a}$ Department of Physics, Adnan Menderes University, Aydn 09010, Turkey

${ }^{b}$ Department of Physics, Bilkent University, Ankara 06800, Turkey.

E-mail: ciraci@fen.bilkent.edu.tr

${ }^{c}$ UNAM-Institute of Materials Science and Nanotechnology, University, Ankara 06800, Turkey

${ }^{d}$ Department of Electrical and Electronic Engineering, Adnan Menderes University, 09100 Aydn, Turkey

${ }^{e}$ Nanotechnology Application and Research Center, Adnan Menderes University, Aydn 09010, Turkey. E-mail: ethem.akturk@adu.edu.tr; Fax: +90 2562135379; Tel: +902562130835

$\dagger$ Electronic supplementary information (ESI) available: Energy bands of patterned structures consisting of a single adatom adsorbed to each periodically repeating $(2 \times 2)$ supercell of h-AlN and h-GaN. The details about the specific hydrogenation geometries, which lead to dynamical instabilities and configurations of approach of $\mathrm{H} 2$ and $\mathrm{O} 2$ molecules, which lead to dissociation and physisorption. See DOI: 10.1039/c8cp02188k
}

were demonstrated based on rigorous phonon calculations. ${ }^{2-4}$ Following the predictions of graphene-like GaN and AlN, which will be denoted as h-GaN and h-AlN in this paper, theoretical studies continued to unveil their diverse features. ${ }^{5-20}$ Attaining outstanding optoelectronic properties of three-dimensional (3D), group III-V compound nitride semiconductors in their $2 \mathrm{D}$ allotropes for future applications in 2D electronics has been the major driving force for the growing research interest in h-GaN and h-AlN.

Recently, h-GaN and h-AlN gained further importance owing to the realization of ultrathin $\mathrm{AlN}$ and GaN in honeycomb structures. Tsipas et $a l^{21}$ demonstrated the epitaxial growth of an ultrathin hexagonal form of AlN on single crystal Ag(111) in 2013. More recently, few monolayer thick flat h-AlN was formed on an $\mathrm{Si}(111)$ surface by molecular beam epitaxy with a lattice constant of $3.08 \AA^{22}$ Also, 2D GaN has been synthesized via graphene encapsulation. ${ }^{23}$ Theoretical and experimental studies show that monolayer h-GaN and h-AlN are indirect, wide band gap semiconductors. They readily form bilayers and multilayers, even h-GaN/h-AlN heterostructures, whereby the electronic and optical properties can be tuned by the number of layers. $^{16-20}$

Other efficient ways to modify the materials and hence to attain diverse physical properties have been the chemisorption of foreign atoms or molecules, substitution of host atoms by 
other atoms (referred also as doping) and creation of patterned vacancies or divacancies in these $2 \mathrm{D}$ structures. ${ }^{24-28}$ The coverage and geometry of the chemisorbed adatoms or molecules are crucial parameters to control the physical properties. Vacancies and divacancies have been shown to be active sites for dissociating molecules like $\mathrm{H}_{2} \mathrm{O}$ and $\mathrm{O}_{2} \cdot{ }^{29}$ Here, one expects that chemical doping or substitution of foreign atoms and creation of vacancies in low (dilute) concentrations can give rise to localized states in the fundamental band gap and resonance states in the band continua. Accordingly, the electronic structures of h-AlN and h-GaN are modified due to acceptors and donors or simply impurity states in the fundamental band gap, the energies of which vary depending on the type of foreign atom and type of vacancy. The localized states are broadened and form impurity bands with increasing coupling among impurities at higher concentrations. Additionally, owing to the unpaired electrons local magnetic moments attribute magnetic properties. Earlier, it has been shown that the band gap is reduced when extended line defects are introduced into the honeycomb structures of h-AlN and h-GaN monolayers. ${ }^{30}$ More recently, theoretical studies have been published about magnetism due to the adsorption of some non-metal atoms to $\mathrm{h}-\mathrm{GaN},{ }^{31}$ the adsorption of $\mathrm{O}$ and $\mathrm{Pt}$ atoms and their clusters on h-AlN, ${ }^{32} \mathrm{Mg}$ doping of $\mathrm{h}-\mathrm{AlN}^{33}$ and $\mathrm{h}-\mathrm{GaN}^{34}$ and $\mathrm{Ga}$ or $\mathrm{N}$ single vacancy charged defects in h-GaN. ${ }^{35}$

In this work, we studied the chemisorption of several adatoms, $\mathrm{H}, \mathrm{C}, \mathrm{N}, \mathrm{O}, \mathrm{F}, \mathrm{Al}, \mathrm{Si}, \mathrm{Ga}, \mathrm{Ge}$, and As, and the physisorption of $\mathrm{H}_{2}$ and $\mathrm{O}_{2}$ molecules on h-AlN and h-GaN, the substitution of C, N, $\mathrm{O}, \mathrm{Mg}, \mathrm{Al}, \mathrm{Si}$ and $\mathrm{Ni}$ atoms into h-AlN and the substitution of $\mathrm{C}$, $\mathrm{N}, \mathrm{O}, \mathrm{Al}, \mathrm{Si}, \mathrm{Cr}, \mathrm{Zn}, \mathrm{Ga}$, and Ge into h-GaN in the low concentration limit. To this end, single adsorbed or substitutional atoms are treated within the supercell geometry to mimic single, isolated dopants. For each adatom the equilibrium binding geometry is determined by optimizing the total energy corresponding to different possible adsorption sites. This way, the effect of several dopants on the atomic, electronic and magnetic properties of both monolayers are unveiled. The stability of specific adatom + substrate structures are tested by performing ab initio molecular dynamics calculations. It is also shown that adatoms modify the atomic structure of the h-GaN bilayer in the heackelite structure by breaking the vertical bonds to lead to changes in electronic structure. Hydrogen and fluorine atoms are exceptions, which were treated in the low, and high concentration limits for different coverage geometries revealing crucial half-metallic and optical properties. Adjacent, as well as separated cation and anion neutral vacancies coexisting in the same supercell, and anti-site defects are also considered.

\section{Method}

First principles calculations were performed using the VASP package $^{36,37}$ on the basis of density functional theory (DFT) within the generalized gradient approximation (GGA). D2-Grimme correction (DFT-D2) $)^{38}$ was used to take van der Waals (vdW) interactions into account. The Perdew-Burke-Ernzerhof (PBE) functionals were used for the exchange-correlation potential, ${ }^{39}$ where the
PAW (Projector Augmented-Wave) potentials were adopted. ${ }^{40,41}$ A cutoff energy of $550 \mathrm{eV}$ for the plane wave basis set and Monkhorst-Pack ${ }^{42}$ mesh of $3 \times 3 \times 1$ for the Brillouin zone integration were employed. Calculations were performed using periodically repeating supercells with a $20 \AA$ vacuum spacing between adjacent monolayers. A $(5 \times 5)$ supercell is found to be suitable to attain negligible couplings between the nearest impurity or defect sites in monolayers. The atomic configurations were optimized by fully relaxing the atomic structures, until the Hellmann-Feynman forces acting on each atom are reduced to less than $0.02 \mathrm{eV} \AA^{-1}$. The convergence of the total energy is achieved until the energy difference between successive iteration steps is less than $10^{-5} \mathrm{eV}$.

Four possible adsorption sites were considered in the calculations: hollow site $(\mathrm{H})$, above the center of the hexagon; bridge site (B), on the midpoint of the cation-anion bonds; top sites $\left(\mathrm{T}_{\mathrm{Al}}\right.$ or $\left.\mathrm{T}_{\mathrm{Ga}}\right)$, on the top of $\mathrm{Al}$ or $\mathrm{Ga}$ atoms; and top sites $\left(\mathrm{T}_{\mathrm{N}}\right)$, on the top of $\mathrm{N}$ atoms. The binding energy $\left(E_{\mathrm{b}}\right)$ of the adatom (A) is calculated from the expression $E_{\mathrm{b}}=E_{\mathrm{T}}[\mathrm{h}-\mathrm{Al} / \mathrm{GaN}]+E_{\mathrm{T}}[\mathrm{A}]-$ $E_{\mathrm{T}}[\mathrm{A}+\mathrm{h}-\mathrm{Al} / \mathrm{GaN}]$, in terms of the total energies (per supercell) of pristine monolayer h-AlN or h-GaN, of the free adatom and of the adatom adsorbed monolayer, respectively. This value includes the energy of the bond between the adatom and monolayers, as well as the deformation induced in the monolayer. $E_{\mathrm{b}}>0$ indicates bonding structure. In the case of the physisorption of a molecule, the physisorption energy $E_{\mathrm{p}}$ is positive, but small and is constituted of predominantly $\mathrm{vdW}$ interactions. The formation energy per cell of a cation-anion vacancy is calculated as $E_{\mathrm{V}}=E_{\mathrm{T}}\left[V_{\mathrm{Al} / \mathrm{Ga}}+V_{\mathrm{N}}+\mathrm{h}-\mathrm{Al} / \mathrm{GaN}\right]-E_{\mathrm{T}}[\mathrm{h}-\mathrm{Al} / \mathrm{GaN}]+\mu_{\mathrm{Al} / \mathrm{Ga}}+\mu_{\mathrm{N}}$, in terms of the total energies of the monolayer including one cation and one anion vacancy, of the bare monolayer and the chemical potentials $(\mu)$ of the cation and anion, respectively. Here, $\mu$ is the atomic chemical potential of each constituent corresponding to its global minimum and is equal to the total energy of the constituent per atom. The formation energy for substitution is calculated using the expression, $E_{\mathrm{s}}=E_{\mathrm{T}}[\mathrm{X}+\mathrm{h}-\mathrm{Al} / \mathrm{GaN}]-E_{\mathrm{T}}[\mathrm{h}-\mathrm{Al} / \mathrm{GaN}]+\mu_{[\mathrm{Al} / \mathrm{Ga} / \mathrm{N}]}-\mu_{[\mathrm{X}]}$, in terms of the total energy of a monolayer with one host atom substituted by an $\mathrm{X}$ atom, of the bare monolayer and the chemical potential of the substituted atoms $(\mathrm{Ga} / \mathrm{Al}$ or $\mathrm{N})$ and substituting $\mathrm{X}$ atom, respectively. Here $E_{\mathrm{V}}>0$ and $E_{\mathrm{s}}>0$ indicate that the formation of vacancies and substitutional defects requires energy.

Since the fundamental band gaps are underestimated by DFT (PBE), we also performed calculations using the HSE06 hybrid functional, ${ }^{43,44}$ constructed by mixing $25 \%$ of the Fock exchange with $75 \%$ of the PBE exchange and $100 \%$ of the PBE correlation energy and the range parameter is set to 0.2 . This way, we aimed to predict fundamental band gaps closer to experimental values. We carried out HSE calculations for pristine and hydrogen covered h-AlN and h-GaN, for which HSE calculations are affordable, since they comprise small numbers of atoms in their unit cell. Having deduced unaltered differences between HSE and PBE band gaps, we were able to interpret the results and tendencies obtained for doped and defected h-AlN and h-GaN comprising $\sim 50$ atoms, for which HSE calculations are not affordable. 
To test the stability of specific adatom + monolayer structures, we carried out finite ab initio molecular dynamics (MD) calculations at $0 \mathrm{~K}$ and $500 \mathrm{~K}$ for $2 \mathrm{ps}$. A Nosé thermostat was used and the kinetic energies were normalized at every 50 steps.

\section{Bare $\mathrm{h}$-AlN and $\mathrm{h}-\mathrm{GaN}$ monolayers}

In order to reveal the effects of doping and defects on the electronic properties we first calculate the optimized structure, energetics and electronic structure of h-AlN and h-GaN monolayers. The monolayers have a planar honeycomb structure; the cohesion of them is maintained mainly by ionic + covalent mixed bonding between nearest $\mathrm{sp}^{2}$-orbitals between the anion and cation, while the planarity is assured by $\pi$-bonding between vertical $\mathrm{p}_{z}$-orbitals. Hence, both h-AlN and h-GaN monolayers are favored with sizeable cohesive energy $E_{\mathrm{c}}=10.56 \mathrm{eV}$ and $E_{\mathrm{c}}=8.29 \mathrm{eV}$ per anion and cation pair with optimized hexagonal lattice constants $a=3.12 \AA$ and $a=3.24 \AA$, respectively. Their formation energies can be retrieved by subtracting the cohesive energy of 3D AlN and GaN in wurtzite structures (both are global minima) from the cohesive energy of h-AlN and h-GaN, which are found to be, $E_{\mathrm{f}}=-1.02 \mathrm{eV}$ and $E_{\mathrm{f}}=-0.71 \mathrm{eV}$, respectively. ${ }^{20}$ Negative values for the formation energies indicate that the monolayers correspond to local minima. That these monolayers are dynamically and thermally stable was demonstrated by phonon calculations at $T=0 \mathrm{~K}$ and finite temperature $a b$ initio molecular dynamics calculations. ${ }^{20}$ Both h-AlN and $\mathrm{h}-\mathrm{GaN}$ are indirect wide band gap, non-magnetic semiconductors; the fundamental band gaps calculated by PBE are $2.92 \mathrm{eV}$ and $2.00 \mathrm{eV}$ respectively. It should be noted that small differences between the present and previous values ${ }^{20}$ originate from different pseudopotentials used in different calculations. ${ }^{4}$ We also calculated the fundamental band gaps of pristine h-AlN and h-GaN with
HSE correction over the PBE results as $4.13 \mathrm{eV}$ and $3.31 \mathrm{eV}$, respectively. Notably, the difference between the HSE and PBE band gaps is found to be in the range of $\sim 1.2-1.3 \mathrm{eV}$. In Fig. 1(a) we present calculated total and atom projected densities of states of bare monolayers for the sake of comparison with those of the defected monolayers.

\section{Doping of $h-A I N$ and $h-G a N$ monolayers}

This section discusses the selected chemisorption of adatoms to h-AlN and h-GaN monolayers, as well as the substitution of host monolayer atoms by foreign atoms $\mathrm{X}$ in the low (dilute) concentration limit, where the coupling between the nearest dopants is negligible. In this limit, we aim to mimic a single dopant on (in) the pristine monolayer. This situation is approximately realized by having a dopant in each $(5 \times 5)$ supercell of the monolayer, which is repeated periodically. The physisorption of $\mathrm{H}_{2}$ and $\mathrm{O}_{2}$ molecules is considered using also the same supercell. Our model of chemical doping and possible adsorption sites on a representative $(5 \times 5)$ supercell is described in Fig. 1(b). These adatoms and substituting atoms are selected by examining earlier works investigating the doping of bulk AlN and GaN. For the monolayer structures, group V and group III elements as well as group IV elements, are found to be interesting from the point of view of structural stability and localized impurity states.

\subsection{Chemical doping}

In order to investigate the effects of chemical doping of selected atoms, we first carried out optimization calculations on four possible adsorption sites in Fig. 1(b) and determined energetically
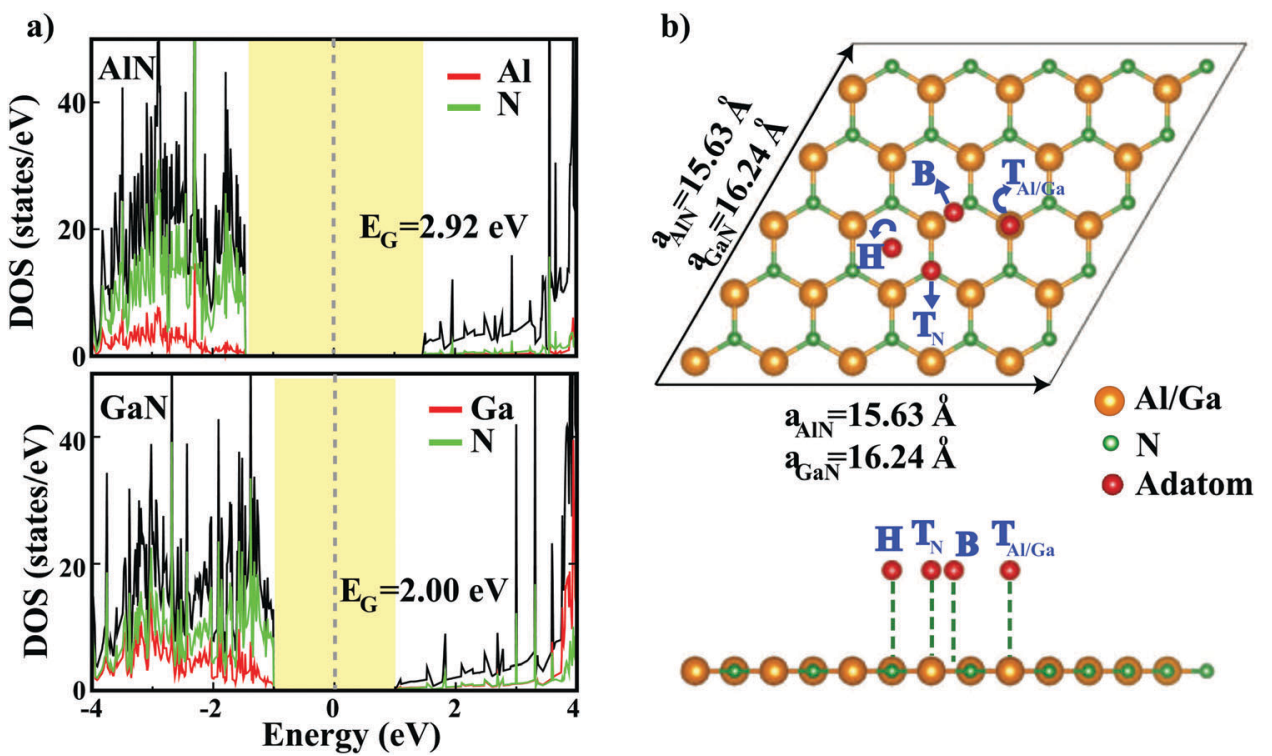

Fig. 1 (a) Total and atom projected densities of states (DOS) of bare h-AIN and h-GaN. Fundamental PBE band gaps are shaded. The zero of band energy is set to the Fermi level. (b) Top and side views of the atomic configuration of the $(5 \times 5)$ supercell of h-AIN or h-GaN monolayers used to treat the doping. Four possible adsorption sites of adatoms and optimized lattice constants of the supercells are shown. 


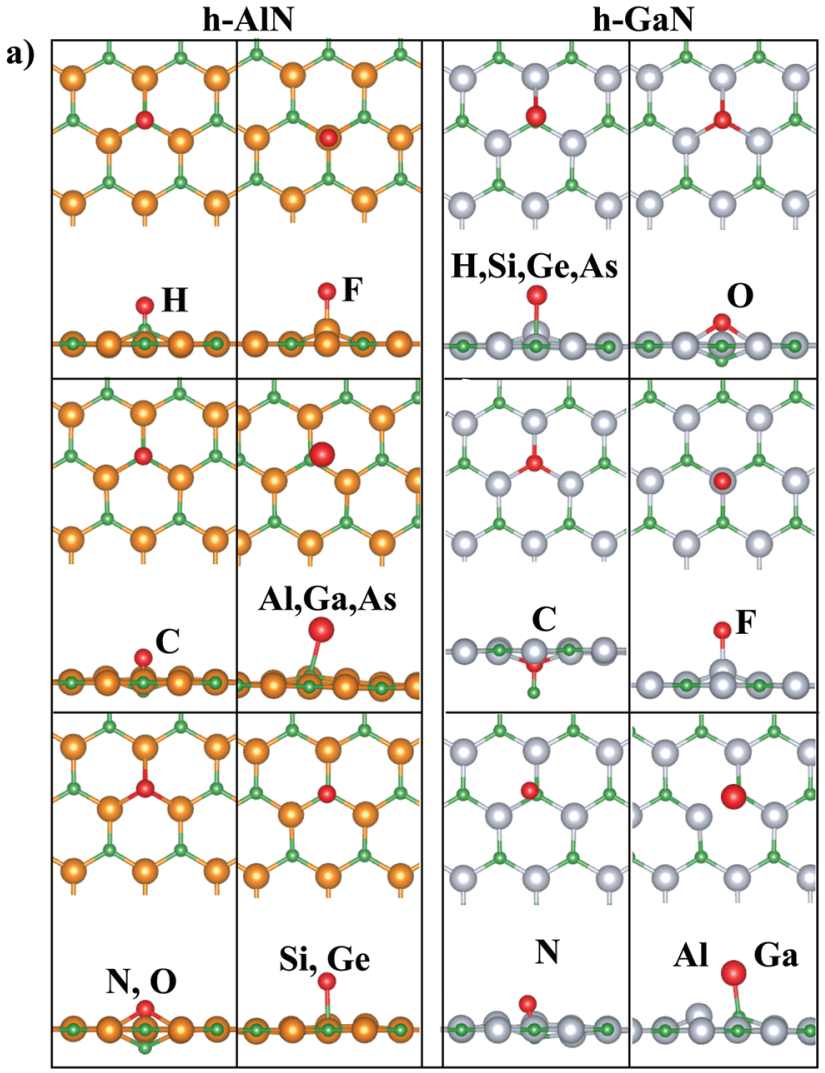

b)

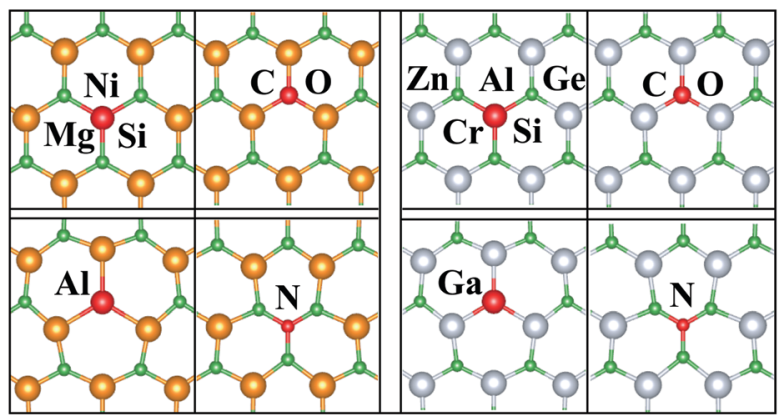

Fig. 2 (a) Top and side views of the optimized atomic configurations of $\mathrm{h}$-AlN and $\mathrm{h}-\mathrm{GaN}$ monolayers with one adatom adsorbed to each $(5 \times 5)$ supercell. Host $\mathrm{Al}, \mathrm{Ga}$, and $\mathrm{N}$ atoms, and foreign adatoms are shown by large orange, large gray, small green and red balls, respectively. (b) Top view of the optimized atomic configuration of $h-A I N$ and $h-G a N$ with one host atom in each $(5 \times 5)$ supercell substituted by selected foreign atoms. Each panel shows only part of the supercell.

the most favorable site for each atom or molecule. Then we calculated the electronic and magnetic structures with adatom induced localized states. In Fig. 2(a), the equilibrium structures of adatoms (H, C, N, O, F, Al, Si, Ga, Ge and As) adsorbed on h-AlN and h-GaN are presented. In Tables 1 and 2, the values calculated for binding energy, magnetic moment and adsorption geometry are given. All adatoms considered in this study form chemical bonds with h-AlN or h-GaN, whereby minute or sizeable deformations at the close proximity of the adatom are induced.

On the h-AlN substrate, the binding energies range from $1.08 \mathrm{eV}$ to $3.95 \mathrm{eV}$. The top of the host $\mathrm{N}$ atom is the equilibrium site for all adatoms treated in this study, except $\mathrm{F}$ that is bound at the top site of the host $\mathrm{Al}$ atom. Adsorbed $\mathrm{N}$ and $\mathrm{O}$ adatoms are bound at the top site of host $\mathrm{N}$ atom by pushing the host down and eventually form an asymmetric dumbbell-like structure. Except for adsorbed $\mathrm{O}$, the rest of the adatoms induce permanent magnetic moments. Overall the features of chemisorbed adatoms adsorbed on h-GaN display similarities to those of h-AlN. On the h-GaN substrate, the top site of the host $\mathrm{N}$ atom is also the equilibrium site of the adatoms $\mathrm{H}, \mathrm{C}, \mathrm{N}, \mathrm{O}$, $\mathrm{Al}, \mathrm{Si}, \mathrm{Ga}, \mathrm{Ge}$ and As. The equilibrium site of the adatom $\mathrm{F}$ is the top of the host Ga. These adatoms form strong chemical bonds with binding energies ranging from $1.19 \mathrm{eV}$ to $3.88 \mathrm{eV}$. The oxygen adatom at the top of the host $\mathrm{N}$ atom pushes it downwards and forms an asymmetric dumbbell-like structure. The adsorbed $\mathrm{C}$ atom also pushes the host $\mathrm{N}$ atom downwards, but it remains in-plane with h-GaN. For both substrates all adatoms are adsorbed at (or near) the top site of the host $\mathrm{N}$ atom; $\mathrm{F}$ being an exception, it is bound to the top site of host metal atoms $\mathrm{Al}$ or $\mathrm{Ga}$.

The contribution of $\mathrm{vdW}$ interaction to the binding is revealed by calculating the binding energy $E_{\mathrm{b}}$ with and without including vdW interaction. The vdW contribution to the $E_{\mathrm{b}}$ ranges between $50 \mathrm{meV}$ and $240 \mathrm{meV}$ per supercell for h-AlN, and $50 \mathrm{meV}$ and $780 \mathrm{meV}$ per supercell for the h-GaN supercell as seen in Tables 1 and 2 .

Among all the binding structures described in Fig. 2(a), the asymmetric dumbbell-like structures formed after the chemical doping of h-AlN and h-GaN by $\mathrm{O}$ and $\mathrm{N}$ adatoms may be prone to instability. The stability of these structures against thermal excitations was tested by performing $a b$ initio MD calculations at $500 \mathrm{~K}$. Snapshots of the atomic configuration in Fig. 3 confirm the stability of these equilibrium adsorption geometries at least at 300-400 K. It should be noted that the dumbell-like structures were found to be essential in the multilayer formation of silicene and germanene (silicite and germanite). ${ }^{45,46}$

The effect of the chemical doping on the electronic structure of bare monolayers at the dilute concentration limit is analyzed in Fig. 4(a) in terms of the total and adatom projected densities of states. The positions of the localized and resonance states relative to the band gap of the bare monolayer are determined

Table 1 Calculated values for an adatom adsorbed to an h-AIN monolayer: Binding energy, $E_{\mathrm{b}}$ (per supercell or per adatom) with/without vdW correction; the height of the adatom from the h-AIN monolayer, $h$; the minimum distance between the adatom and host atom of h-AIN, $d$; and the magnitude of local magnetic moment $\mu$ (per supercell)

\begin{tabular}{llllll}
\hline Adatom $(\mathrm{A})$ & Site & $E_{\mathrm{b}}(\mathrm{eV})$ & $h(\AA)$ & $d(\AA)$ & $\mu\left(\mu_{\mathrm{B}}\right)$ \\
\hline $\mathrm{H}$ & $\mathrm{T}_{\mathrm{N}}$ & $1.08 / 1.03$ & 1.64 & 1.05 & 1.00 \\
$\mathrm{C}$ & $\mathrm{T}_{\mathrm{N}}$ & $3.44 / 3.33$ & 1.10 & 1.45 & 2.00 \\
$\mathrm{~N}$ & $\mathrm{~T}_{\mathrm{N}}$ & $2.59 / 2.49$ & 0.90 & 1.58 & 1.00 \\
$\mathrm{O}$ & $\mathrm{T}_{\mathrm{N}}$ & $3.95 / 3.88$ & 0.98 & 1.68 & 0 \\
$\mathrm{~F}$ & $\mathrm{~T}_{\mathrm{Al}}$ & $3.51 / 3.41$ & 2.26 & 1.71 & 1.00 \\
$\mathrm{Al}$ & $\sim \mathrm{T}_{\mathrm{N}}$ & $1.31 / 1.13$ & 2.41 & 2.10 & 1.00 \\
$\mathrm{Si}$ & $\sim \mathrm{T}_{\mathrm{N}}$ & $2.03 / 1.89$ & 2.11 & 1.97 & 2.00 \\
$\mathrm{Ga}$ & $\sim \mathrm{T}_{\mathrm{N}}$ & $1.12 / 0.88$ & 2.37 & 2.38 & 1.00 \\
$\mathrm{Ge}$ & $\sim \mathrm{T}_{\mathrm{N}}$ & $1.66 / 1.46$ & 2.26 & 2.19 & 2.00 \\
$\mathrm{As}$ & $\sim \mathrm{T}_{\mathrm{N}}$ & $1.26 / 1.05$ & 2.10 & 2.10 & 1.00 \\
$\mathrm{H}_{2}$ & $\mathrm{~B}$ & $0.05 /-$ & 2.43 & 2.70 & 0 \\
$\mathrm{O}_{2}$ & $\mathrm{~T}_{\mathrm{Al}}$ & $0.08 /-$ & 2.91 & 3.08 & 2.00
\end{tabular}


Table 2 Calculated values for an adatom adsorbed to an $\mathrm{h}$-GaN monolayer: Binding energy, $E_{\mathrm{b}}$ (per supercell or per adatom) with/without vdW correction; the height of the adatom from the $\mathrm{h}-\mathrm{GaN}$ monolayer, $h$; the minimum distance between the adatom and host atom of $\mathrm{h}-\mathrm{GaN}, d$; and the magnitude of local magnetic moment $\mu$ (per supercell)

\begin{tabular}{lllrll}
\hline Adatom $(\mathrm{A})$ & Site & $E_{\mathrm{b}}(\mathrm{eV})$ & $h(\AA)$ & $d(\AA)$ & $\mu\left(\mu_{\mathrm{B}}\right)$ \\
\hline $\mathrm{H}$ & $\mathrm{T}_{\mathrm{N}}$ & $1.72 / 1.67$ & 1.57 & 1.04 & 0 \\
$\mathrm{C}$ & $\mathrm{T}_{\mathrm{N}}$ & $3.88 / 3.79$ & -0.53 & 1.33 & 2.00 \\
$\mathrm{~N}$ & $\sim \mathrm{T}_{\mathrm{N}}$ & $1.84 / 1.75$ & 1.16 & 1.54 & 1.00 \\
$\mathrm{O}$ & $\mathrm{T}_{\mathrm{N}}$ & $3.05 / 2.99$ & 1.06 & 1.69 & 0 \\
$\mathrm{~F}$ & $\mathrm{~T}_{\mathrm{Ga}}$ & $2.91 / 2.13$ & 2.44 & 1.86 & 1.00 \\
$\mathrm{Al}$ & $\sim \mathrm{T}_{\mathrm{N}}$ & $1.55 / 1.37$ & 2.57 & 1.98 & 1.00 \\
$\mathrm{Si}$ & $\mathrm{T}_{\mathrm{N}}$ & $1.96 / 1.81$ & 2.14 & 1.95 & 2.00 \\
$\mathrm{Ga}$ & $\sim \mathrm{T}_{\mathrm{N}}$ & $1.26 / 1.01$ & 2.63 & 2.26 & 1.00 \\
$\mathrm{Ge}$ & $\mathrm{T}_{\mathrm{N}}$ & $1.60 / 1.41$ & 2.23 & 2.21 & 2.00 \\
$\mathrm{As}$ & $\mathrm{T}_{\mathrm{N}}$ & $1.19 / 0.99$ & 2.28 & 2.09 & 1.00 \\
$\mathrm{H}_{2}$ & $\mathrm{~T}_{\mathrm{N}}$ & $0.06 /-$ & 2.36 & 2.61 & 0 \\
$\mathrm{O}_{2}$ & $\mathrm{~T}_{\mathrm{Ga}}$ & $0.07 /-$ & 3.02 & 3.22 & 2.00 \\
\hline
\end{tabular}

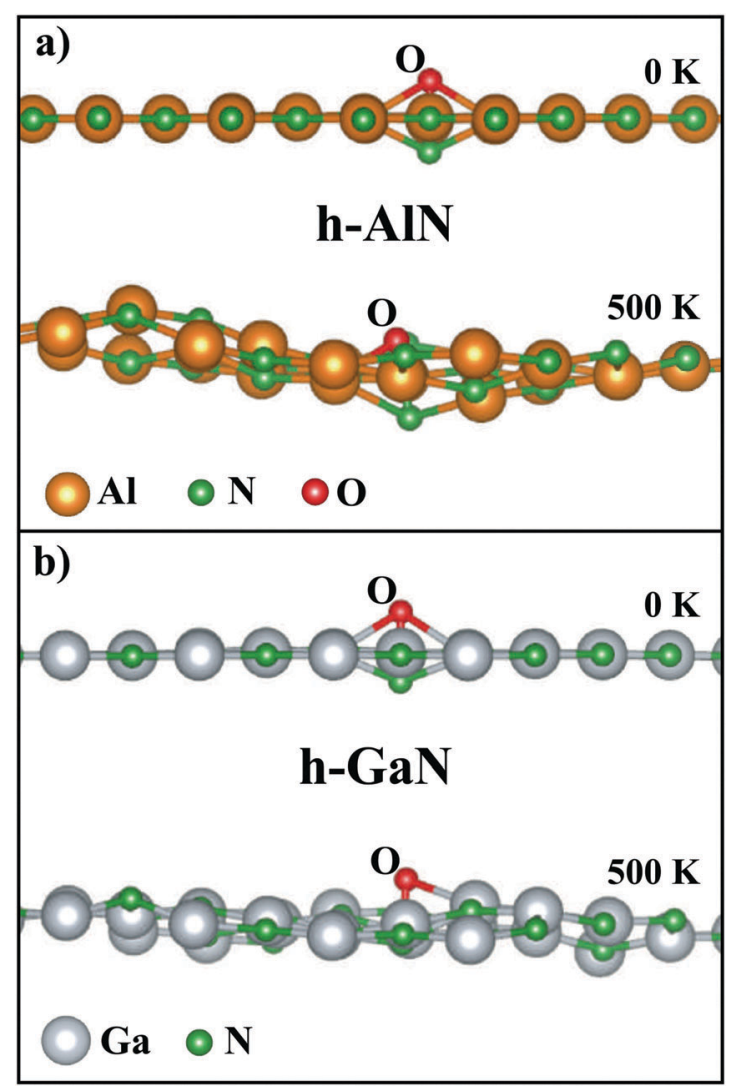

Fig. 3 (a) Snapshots of the atomic configuration of the $\mathrm{O}$ adatom adsorbed to each $(5 \times 5)$ supercell of $\mathrm{h}$-AIN taken at $0 \mathrm{~K}$ and $500 \mathrm{~K}$ in ab initio molecular dynamics calculations; (b) the same for the $\mathrm{h}-\mathrm{GaN}$ substrate.

from the density of states projected to a cation-anion bond of the monolayer, which is farthest to the adatom in the supercell. The fundamental band gap of the bare monolayer determined therefrom is shaded. In Fig. 4(b) all localized states derived from the chemical doping of adatoms are displayed in the fundamental band gap of pristine monolayers. The $\mathrm{Ga}$ adatom constitutes shallow states close to the valence band edge of
h-GaN; however, it does not have any impurity state in the band gap of h-AlN. In contrast, the Ge adatom does not form localized states in the band gap of h-GaN, while it has shallow donor states near the conduction band edge of h-AlN. The hydrogen adatom introduces donor states for both h-AlN and $\mathrm{h}-\mathrm{GaN}$. The fundamental band gap of the monolayers becomes free of the localized states upon the adsorption of an $\mathrm{O}$ adatom. Other details related with the chemical doping of the monolayers can be retrieved from Fig. 4.

Another effect of chemical doping is the local magnetization induced in h-AlN and h-GaN monolayers. The adatom induced magnetic state and resulting net magnetic moments per supercell are determined by the optimization of the atomic and magnetic structures, and confirmed by the total density of states using fine mesh. In general, while adatoms from group III and V of the Periodic Table induced $1.0 \mu_{\mathrm{B}}$ per cell magnetic moment, adatoms from group IV induced $2.0 \mu_{\mathrm{B}}$ per cell magnetic moment after adsorption. The fluorine (F) adatom of group VII adsorbed on the top of the metal atoms induces also $1.0 \mu_{\mathrm{B}}$ per cell local magnetic moment. The pristine h-AlN monolayer is nonmagnetic, but acquires magnetization upon the adsorption of all adatoms except $\mathrm{O}$ atoms. The spindegeneracy is broken; $\mathrm{Al}, \mathrm{C}, \mathrm{F}, \mathrm{N}$ and $\mathrm{Si}$ adatoms adsorbed on the h-AlN monolayer give rise to localized states of one spindirection overlapping with the Fermi level, and localized states of the other spin-direction above and below the Fermi level. For h-GaN, Al, C, F, N, Si and As display a similar behavior.

When the coupling between these localized states becomes significant at high adatom coverage, the localized states start to form bands in the gap. Then, the above behavior may lead to half-metallicity, where the band of one spin-direction is metallic, while that of the opposite spin-direction becomes a semiconductor. This way a half-metal can transport only electrons of one spin-direction. Half-metallic behavior depends on the coverage geometry of specific adatoms. Here we consider only specific adatoms and explored whether they can lead to half-metallicity when they covered a single side of the h-AlN and h-GaN monolayers to form a $(2 \times 2)$ mesh. This is a high concentration, even ordered decoration of adatoms like a compound. We found that the $\mathrm{F}$ adatom adsorbed on h-AlN and h-GaN monolayers to form a periodic $(2 \times 2)$ mesh attained a half-metallic band structure. While spin-up bands constitute an indirect band gap semiconductor, two spin-down bands, which are degenerate at the center of the Brillouin zone, are half-filled and hence become metallic as shown in Fig. 5 . Accordingly the net magnetic moment per cell is $1.0 \mu_{\mathrm{B}}$. Notably, integer values of $\mu$ are characteristic of half-metals.

In addition to the $\mathrm{F}$ adatom, we also considered $\mathrm{Al}, \mathrm{C}, \mathrm{N}$ and Si adsorbed to h-AlN and C, N, Si and As adsorbed to h-GaN forming periodic $(2 \times 2)$ meshes on both monolayers. The band structures of these systems presented in ESI, $\dagger$ Fig. S1 suggest that $\mathrm{Al}, \mathrm{C}, \mathrm{N}$ and $\mathrm{Si}$ adsorbed on h-AlN forming a periodic $(2 \times 2)$ mesh are semiconductors for both spin-directions. A similar situation is also found for $\mathrm{C}, \mathrm{N}, \mathrm{Si}$ and As adatoms adsorbed on h-GaN. However, some of these magnetic semiconductors have a narrow band gap for one spin-direction and 
a)
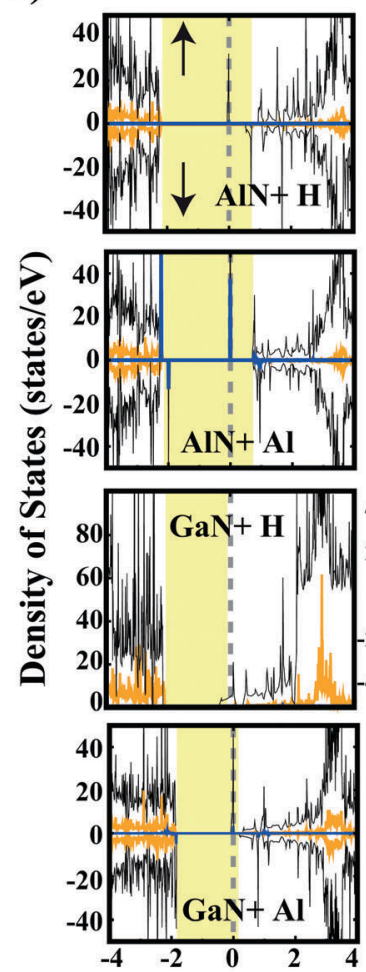

Gân+C
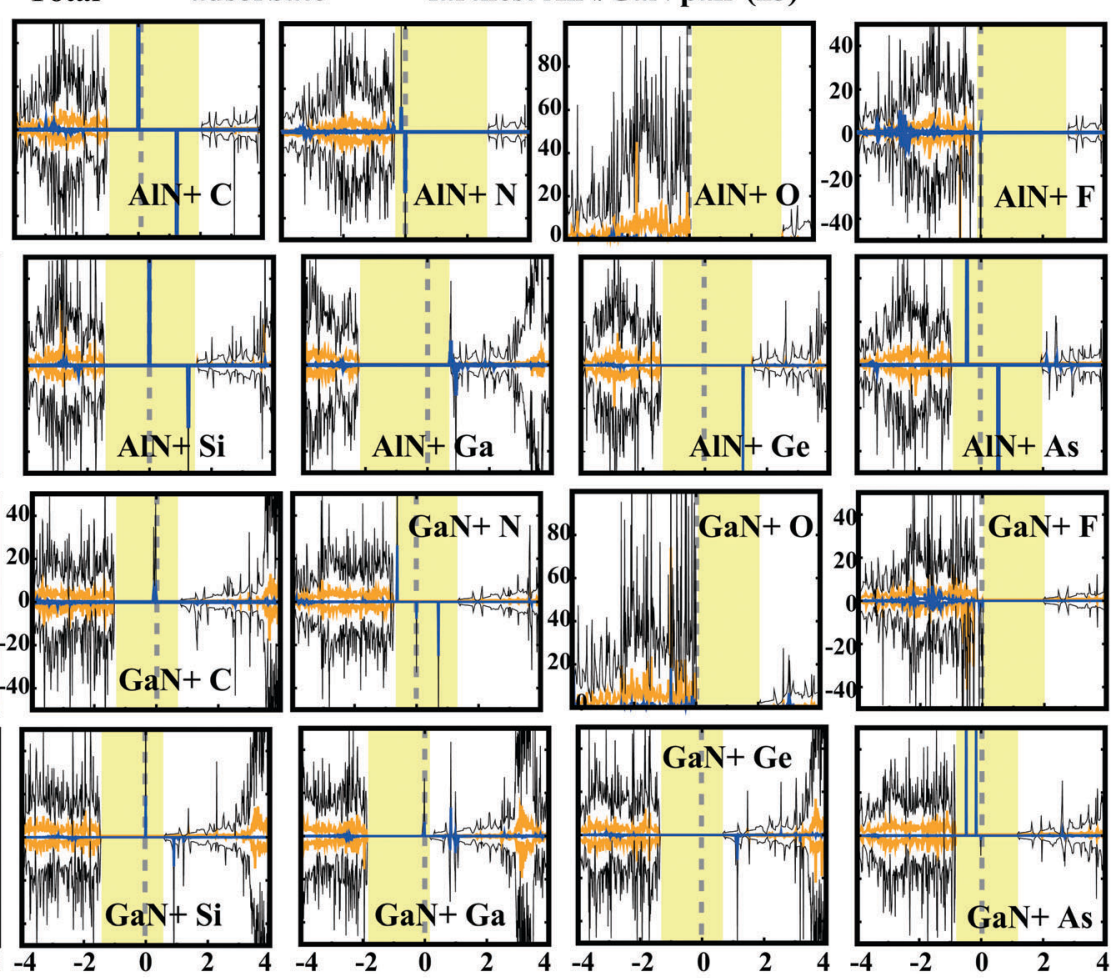

Energy (eV)
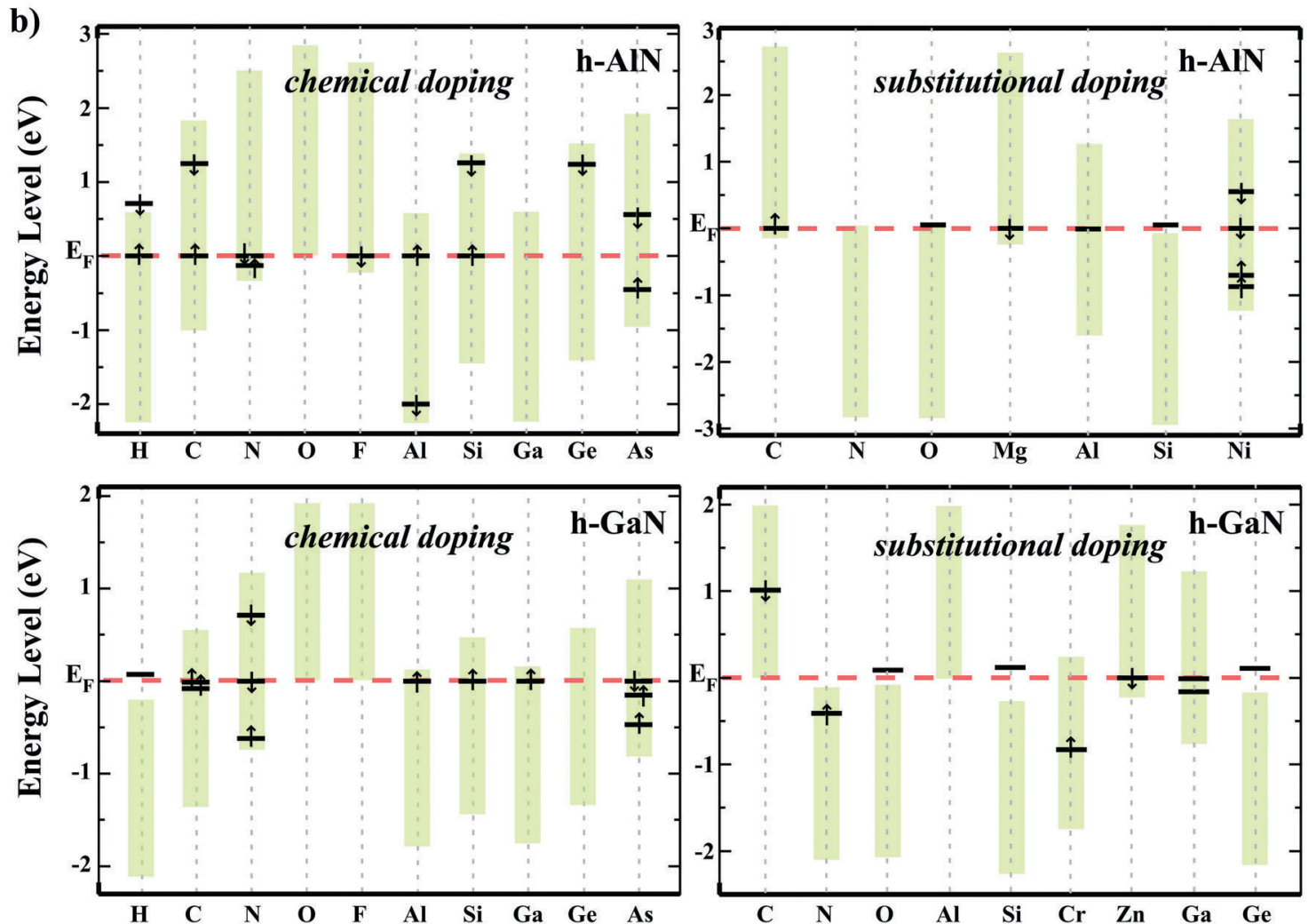

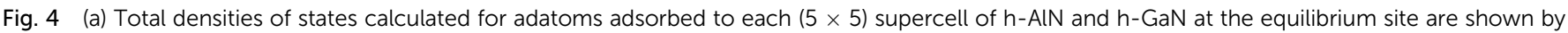

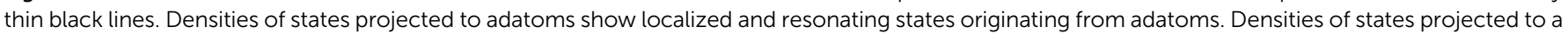

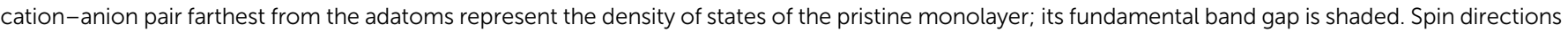

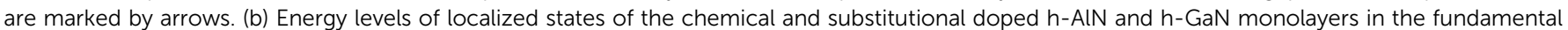

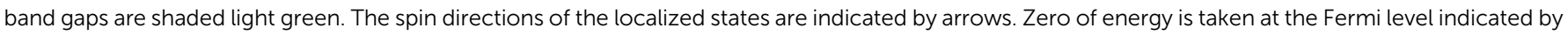
dashed lines. 

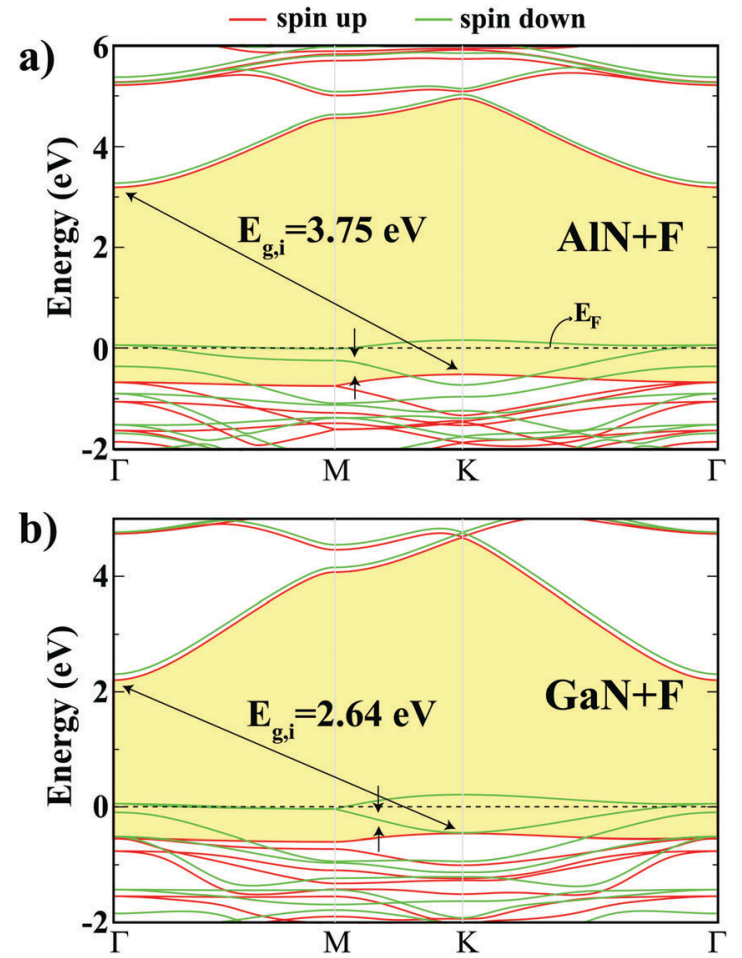

Fig. 5 Energy bands of $\mathrm{F}$ adsorbed to each $(2 \times 2)$ supercell of monolayers having significant coupling with nearest host atoms. (a) AIN + F. (b) GaN + F. Zero of energy is taken at the Fermi Level. Spin-polarized energy bands display half-metallic properties.

can easily be photoexcited and change into metals with low carrier concentration in the conduction band. Those having a very narrow band gap can also acquire a significant number of carriers at room temperature. Eventually, when excited, these magnetic semiconductors can operate as half-metals. It should be noted that these half-metals can be important only if their Curie temperatures are high enough to allow spintronics applications.

$\mathrm{H}_{2}$ and $\mathrm{O}_{2}$ molecules are also physisorbed on h-AlN and h-GaN monolayers through vdW interaction. The binding energies are weak and range from $60 \mathrm{meV}$ to $80 \mathrm{meV}$, and their distance to the monolayer is large. Earlier, $\mathrm{H}_{2}$ was found to be physisorbed to h-GaN with an energy of $150 \mathrm{meV}$, but $\mathrm{O}_{2}$ was unbound. ${ }^{47}$ Because of the weak vdW interaction between $\mathrm{O}_{2}$ and h-AlN and h-GaN substrates the magnetic moment of the free $\mathrm{O}_{2}$ is maintained after the physisorption. In forthcoming sections we show that these molecules can engage in strong interaction at the vacancy site leading to dissociation.

Apart from important modifications of chemical doping in the atomic and electronic structure of $\mathrm{h}-\mathrm{GaN}$, another effect of specific adatoms on the bilayer of h-GaN is worth noting. Two h-GaN monolayers form normally planar bilayers, where host $\mathrm{Ga}$ atoms of one monolayer are located over host $\mathrm{N}$ atoms of the other monolayer and vice versa having a distance larger than the normal Ga-N bond distance. This way, the bilayer is held through vdW attraction. However, the planar bilayer can form a heackelite structure with the gain of a minute energy, whereby some vertical Ga-N pairs form weak covalent bonds, while the a)
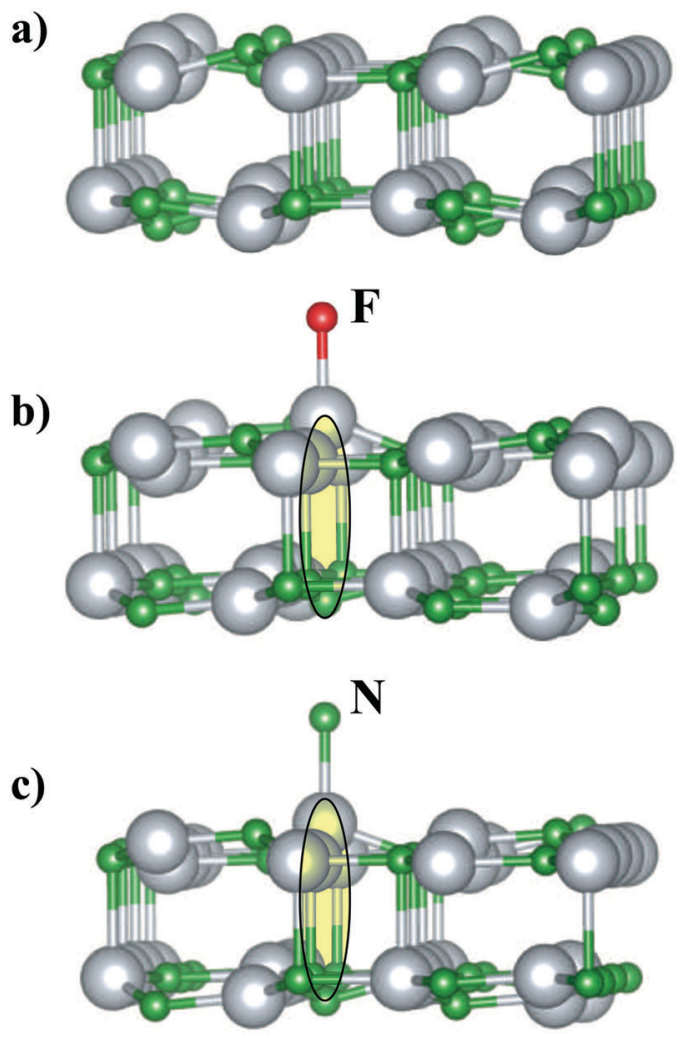

Fig. 6 (a) Bilayer of GaN in heackelite structure with specific vertical $\mathrm{Ga}-\mathrm{N}$ bonds; (b) opening of vertical $\mathrm{Ga}-\mathrm{N}$ bonds upon adsorption of an $\mathrm{F}$ adatom; (c) the same for an $\mathrm{N}$ adatom.

others go away further from each other ${ }^{12,20}$ as described in Fig. 6(a). This structural transition from planar bilayer to heackelite bilayer results in the change of the fundamental band gap from indirect to direct. The adsorption of $\mathrm{F}$ and $\mathrm{N}$ atoms on the heackelite structure causes the vertical Ga-N bond to break as shown in Fig. 6(b and c). This particular structural change results in changes in the electronic structure. Bond breaking occurs due to the charge transfer from the vertical Ga-N bond to the adatom.

\subsection{Substitutional doping}

In the past the substitutional doping of $3 \mathrm{D}$ AlN and $\mathrm{GaN}$ wurtzite crystals by foreign atoms $(\mathrm{Mg}, \mathrm{Ni}, \mathrm{Si}, \mathrm{Ge}, \mathrm{Cr})$ was investigated extensively. In view of the earlier research on the

Table 3 Calculated values for the host atoms of the h-AIN monolayer substituted by selected foreign atoms. Formation energy of substitution $E_{\mathrm{s}}$ (per supercell); the average distance between the substituting atom and nearest host atoms of $\mathrm{h}$-AlN, $d$; the magnitude of local magnetic moment $\mu$ (per supercell); optimized lattice constant of the $(5 \times 5)$ supercell, a

\begin{tabular}{llrlll}
\hline Atom $(\mathrm{A})$ & Site & $E_{\mathrm{S}}(\mathrm{eV})$ & $d(\AA)$ & $\mu\left(\mu_{\mathrm{B}}\right)$ & $a(\AA)$ \\
\hline $\mathrm{C}$ & $\mathrm{N}$ & 4.62 & 1.90 & 1.00 & 15.68 \\
$\mathrm{~N}$ & $\mathrm{Al}$ & 7.68 & 1.44 & 0 & 15.47 \\
$\mathrm{O}$ & $\mathrm{N}$ & -1.06 & 1.84 & 0 & 15.63 \\
$\mathrm{Mg}$ & $\mathrm{Al}$ & 3.32 & 1.95 & 1.00 & 15.69 \\
$\mathrm{Al}$ & $\mathrm{N}$ & 8.54 & 2.32 & 0 & 15.85 \\
$\mathrm{Si}$ & $\mathrm{Al}$ & 1.58 & 1.70 & 0 & 15.60 \\
$\mathrm{Ni}$ & $\mathrm{Al}$ & 5.33 & 1.83 & 3.00 & 15.63
\end{tabular}


Table 4 Calculated values for the host atoms of the h-GaN monolayer substituted by selected foreign atoms. Formation energy of substitution, $E_{\mathrm{s}}$ (per supercell); the average distance between the substituting atom and nearest host atoms of $\mathrm{h}-\mathrm{GaN}$, $d$; the magnitude of local magnetic moment $\mu$ (per supercell); optimized lattice constant of the $(5 \times 5)$ supercell, a

\begin{tabular}{llrlll}
\hline Atom $(\mathrm{A})$ & Site & $E_{\mathrm{s}}(\mathrm{eV})$ & $d(\AA)$ & $\mu\left(\mu_{\mathrm{B}}\right)$ & $a(\AA)$ \\
\hline $\mathrm{C}$ & $\mathrm{N}$ & 3.33 & 1.93 & 1.00 & 16.26 \\
$\mathrm{~N}$ & $\mathrm{Ga}$ & 5.91 & 1.43 & 1.56 & 16.11 \\
$\mathrm{O}$ & $\mathrm{N}$ & -1.29 & 1.94 & 0 & 16.27 \\
$\mathrm{Al}$ & $\mathrm{Ga}$ & -1.74 & 1.81 & 0 & 16.24 \\
$\mathrm{Si}$ & $\mathrm{Ga}$ & -0.59 & 1.70 & 0 & 16.24 \\
$\mathrm{Cr}$ & $\mathrm{Ga}$ & 1.31 & 1.86 & 3.00 & 16.24 \\
$\mathrm{Zn}$ & $\mathrm{Ga}$ & 2.30 & 1.90 & 1.00 & 16.27 \\
$\mathrm{Ga}$ & $\mathrm{N}$ & 4.37 & 2.26 & 0 & 16.43 \\
$\mathrm{Ge}$ & $\mathrm{Ga}$ & 1.41 & 1.83 & 0 & 16.26 \\
& & & & &
\end{tabular}

substitutional doping of 3D AlN and GaN, here we consider specific foreign atoms to substitute cation and anion atoms of h-AlN and h-GaN. For the h-AlN monolayer, the host $\mathrm{N}$ atom is substituted by $\mathrm{C}, \mathrm{O}$ and $\mathrm{Al}$ foreign atoms; the $\mathrm{Al}$ host atom is substituted by $\mathrm{N}, \mathrm{Mg}, \mathrm{Si}$ and Ni foreign atoms. For the h-GaN monolayer, the host $\mathrm{N}$ atom is substituted by $\mathrm{C}, \mathrm{O}$ and $\mathrm{Ga}$ foreign atoms; the host $\mathrm{Ga}$ atom is substituted by $\mathrm{N}, \mathrm{Al}, \mathrm{Si}, \mathrm{Cr}$, $\mathrm{Zn}$ and Ge foreign atoms. Relevant values, like the formation energy of substitution $E_{\mathrm{s}}$, magnetic moment and optimized bond lengths and lattice constant are presented in Tables 3 and 4 . Calculations were performed by using a $(5 \times 5)$ supercell. The optimized atomic configurations of substitutional doping of h-AlN and h-GaN are shown in Fig. 2(b).

$\mathrm{Al}(\mathrm{N})$ substituting $\mathrm{N}(\mathrm{Al})$ in h-AlN and $\mathrm{Ga}(\mathrm{N})$ substituting $\mathrm{N}(\mathrm{Ga})$ in $\mathrm{h}-\mathrm{GaN}$ causes local reconstructions due to size differences between atoms. Substituting foreign atoms $\mathrm{C}, \mathrm{Mg}$, and $\mathrm{Ni}$ in h-AlN, and $\mathrm{C}, \mathrm{N}, \mathrm{Cr}$, and $\mathrm{Zn}$ in h-GaN induces a magnetic moment. In particular, transition metal atoms $\mathrm{Ni}$ and Cr induce high magnetic moments when they substitute cations in h-AlN and h-GaN, respectively. Specific foreign atoms, such as $\mathrm{O}$ in h-AlN and $\mathrm{O}, \mathrm{Al}$ and $\mathrm{Si}$ in h-GaN, lead to negative formation energy of substitution, $E_{\mathrm{s}}$, indicating the fact that related substitution processes are exothermic. On the contrary,

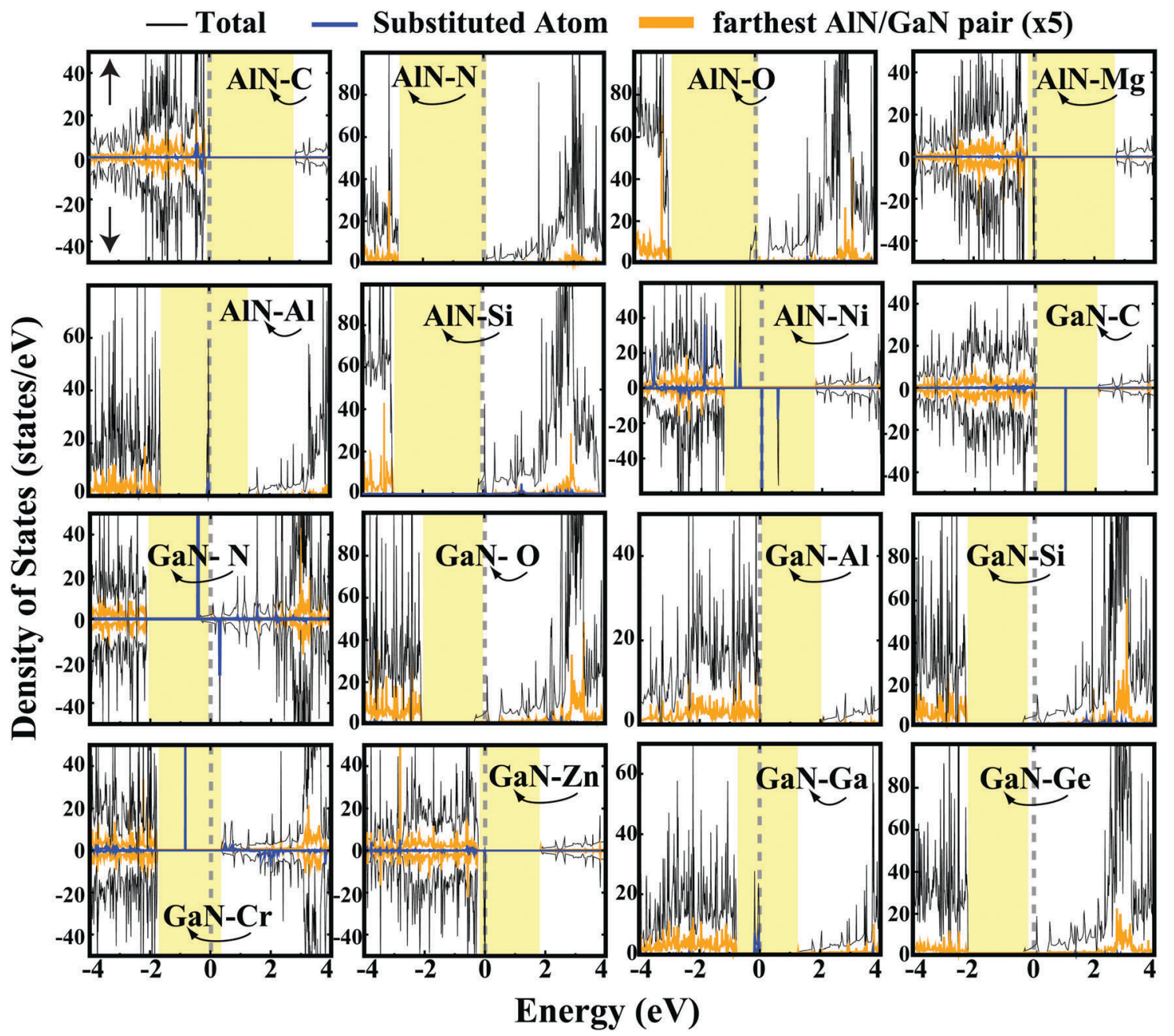

Fig. 7 Total density of states (TDOS) calculated for a foreign atom substituting one host atom in each ( $5 \times 5)$ supercell of $h$-AIN and $h$-GaN as indicated by arrows. Density of states projected to the substituting foreign atom adatom shows localized and resonating states. Density of states projected to a cation-anion pair farthest from the adatom corresponds to the pristine monolayer; its fundamental band gap is shaded. Zero of energy is set at the Fermi level. 
cases with $E_{s}>0$ correspond to an endothermic reaction. Expectantly, the highest $E_{\mathrm{S}}$ values are attained for $\mathrm{Al}$ substitution of the host $\mathrm{N}$ atom in h-AlN and Ga substitution of the host $\mathrm{N}$ atom in the h-GaN monolayer.

The analysis of the total and projected densities of states related with the substitutional doping is summarized in Fig. 7. The h-AlN monolayer remains nonmagnetic, when $\mathrm{Al}(\mathrm{N})$ host atoms are substituted by $\mathrm{N}(\mathrm{Al})$ atoms. If a $\mathrm{N}$ foreign atom substitutes an $\mathrm{Al}$ host, the band gap becomes free of localized states. In the reverse situation, the Fermi level is pinned by localized states at the midgap. Similarly, h-GaN, which is substitutionally doped by foreign $\mathrm{Ga}$ atom is nonmagnetic and has a Fermi level pinned by the localized states near the midgap. On the contrary, host Ga substituted by $\mathrm{N}$ in h-GaN gives rise to a magnetic moment and has localized states below the edge of the conduction band. When the $\mathrm{N}$ host atom is substituted by $\mathrm{O}$ in h-AlN and h-GaN, donor states can occur near the edge of the conduction band. Upon substitutional doping of the host $\mathrm{Al}$ or $\mathrm{Ga}$ atom by a foreign Si atom, both h-AlN and h-GaN remain nonmagnetic and have donor states below the edge of their conduction band. When the host $\mathrm{N}$ atom of h-GaN is substituted by $\mathrm{C}$, h-GaN attains magnetization and has an empty localized state at the midgap. In a specific and regular concentration of these atoms the doped monolayer may gain half-metallic behavior because of coupling of dopants at high concentration. Briefly, when doped substitutionally, h-AlN and h-GaN semiconductors can attain magnetic properties and have filled or empty localized states in the fundamental band gap.

\section{Vacancy and anti-site defects}

Here we consider two types of vacancy defects in h-AlN and h-GaN monolayers: (i) a divacancy (nearest two vacancies) is created by removing the nearest cation (metal) and anion. This defect is denoted by $V_{\mathrm{N}}$. (ii) Two vacancies are created by removing the farthest cation and anion in the supercell, which is specified by $V_{\mathrm{F}}$. Another defect is an anti-site, where one cation exchanges its position with the nearest anion. All these defects in a polar semiconductor give rise to local deformations as shown in Fig. 8.
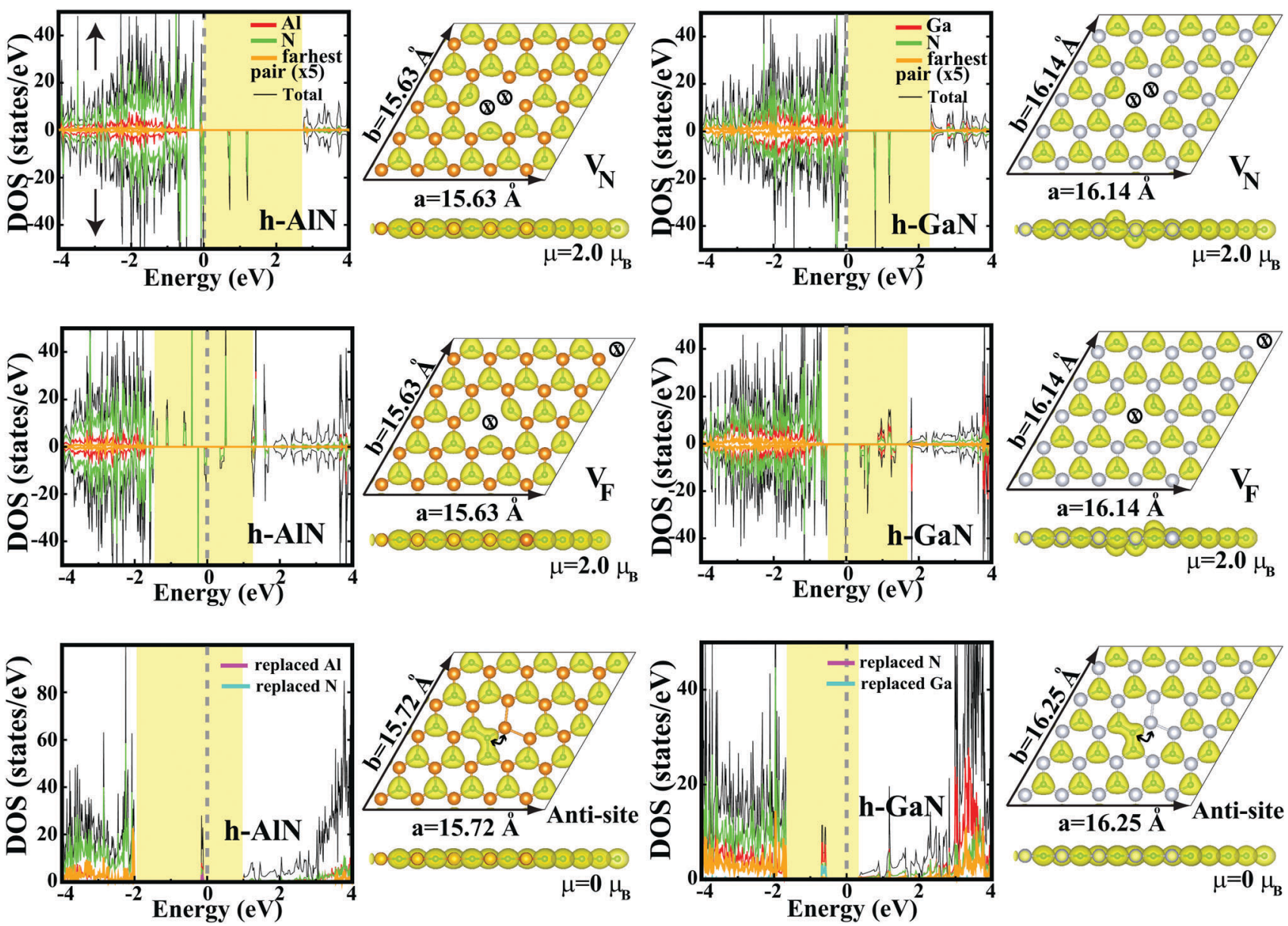

Fig. 8 Nearest cation-anion vacancy, i.e. divacancy $V_{N}$; separated cation and anion vacancy $V_{F}$ and anti-site defects where the nearest cation and anion are exchanged in each $(5 \times 5)$ supercell of $\mathrm{h}$-AIN and $\mathrm{h}$-GaN. Left panels: total density of states and density of states projected to the anion/cation which are adjacent to the vacant site. Band gap of bare monolayer is shaded. Zero of energy is set at the Fermi level. Right panels: top and side views of optimized atomic configuration with isosurfaces of the total charge density in a $(5 \times 5)$ supercell. Removed atoms are indicated by $(x)$. Magnetic moments (per supercell) are also given. 
Owing to the removed cation and anion in vacancy defects, each supercell attains a permanent magnetic moment of $\mu=2.0 \mu_{\mathrm{B}}$ in both h-AlN and h-GaN. However, an anti-site is nonmagnetic like a pristine monolayer. The formation energy of a vacancy defect or anti-site is found to be positive, namely that energy is required to form the defect. The formation energies of $V_{\mathrm{N}}, V_{\mathrm{F}}$ and anti-site are $10.18 \mathrm{eV}, 13.32 \mathrm{eV}$ and $10.12 \mathrm{eV}$, respectively for h-AlN. The calculated formation energies of these defects in h-GaN are $6.46 \mathrm{eV}, 9.48 \mathrm{eV}$ and $6.13 \mathrm{eV}$, respectively. These values are comparable with a recent study performed within LDA. ${ }^{48}$ Notably, $E_{\mathrm{VN}}<E_{\mathrm{VF}}$ due to less bonds being broken to create a $V_{\mathrm{N}}$ in pristine monolayers. Like other defects, analysis of total and projected densities of states reveals filled and empty states localized in the fundamental band gap of h-AlN and h-GaN as described in Fig. 8. The electronic structure of $V_{\mathrm{F}}$ has the potential of forming half-metallic behavior for a specific
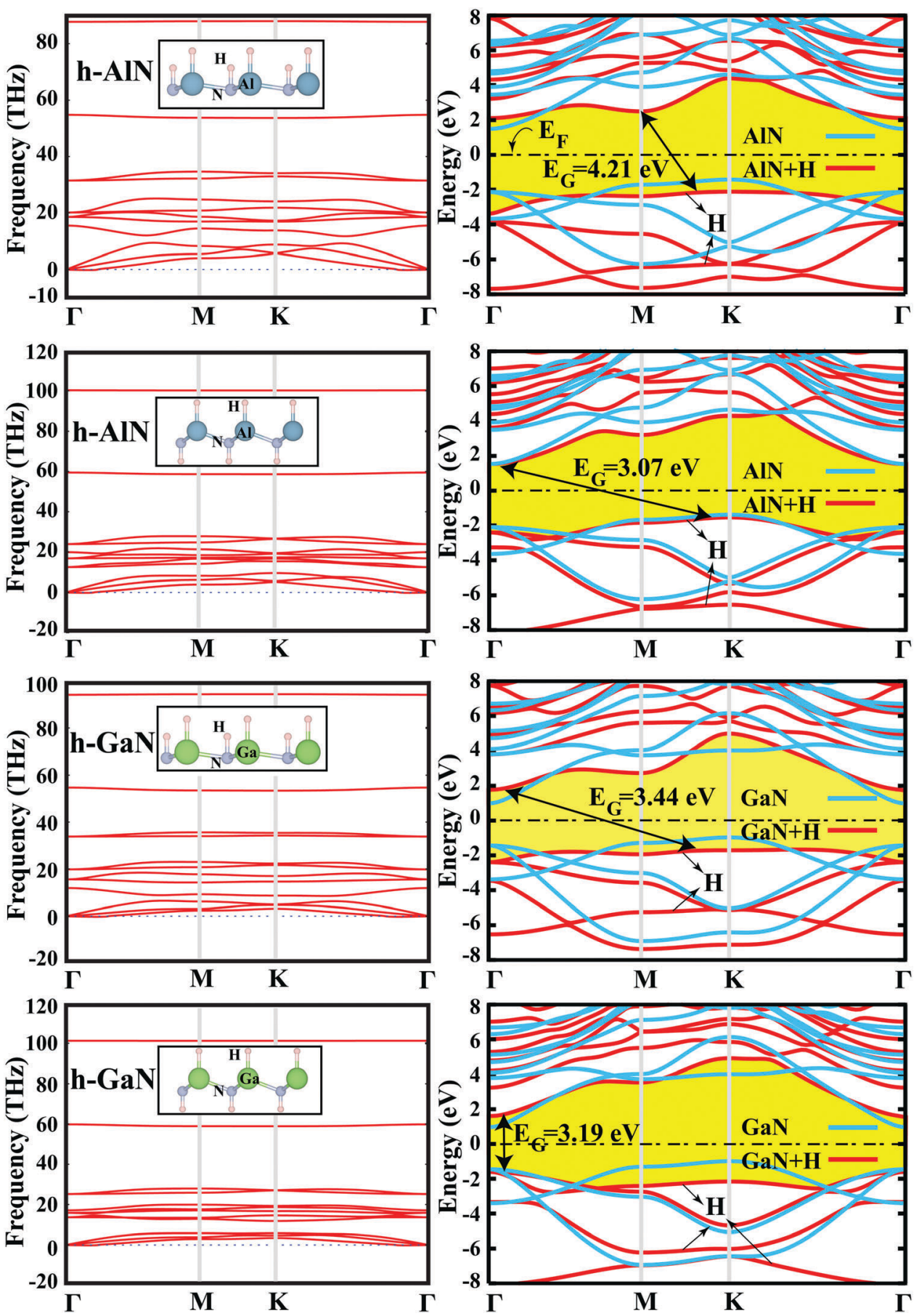

Fig. 9 Left panels: Optimized atomic configurations and corresponding phonon dispersions of single-sided (S-H) and double-sided (DS-H) hydrogenated h-AIN and h-GaN. Right panels: Energy band structures. The fundamental band gaps are shaded. The bands of bare monolayers are shown by light lines. 
Table 5 Values calculated by using PBE for single-sided (S-H) and double-sided (DS-H) hydrogenation of h-AIN and h-GaN monolayers: lattice constant a; buckling height $\Delta$; bond distance between metal ( $M$ or cation) atoms and nitrogen $d_{M-N}$; bond distance between metal $(M)$ atoms and hydrogen $d_{M-H}$; bond distance between nitrogen and hydrogen $d_{\mathrm{N}-\mathrm{H}}$; average binding energy of free $\mathrm{H}$ atom $E_{\mathrm{b}}$; band gap $E_{\mathrm{g}}$ (i: indirect, d: direct gap). HSE corrected band gaps are shown in parentheses

\begin{tabular}{llllllll}
\hline System & $a(\AA)$ & $\Delta(\AA)$ & $d_{\mathrm{M}-\mathrm{N}}(\AA)$ & $d_{\mathrm{M}-\mathrm{H}}(\AA)$ & $d_{\mathrm{N}-\mathrm{H}}(\AA)$ & $E_{\mathrm{c}}(\mathrm{eV}$ per atom) & $E_{\mathrm{g}}(\mathrm{eV})$ \\
\hline $2^{*} \mathrm{~h}-\mathrm{AlN}$ & & & & & & & \\
S-H & 3.30 & 0.29 & 1.93 & 1.61 & 1.06 & 7.20 & 7.82 \\
DS-H & 3.14 & 0.68 & 1.94 & 1.57 & 1.03 & & 3.07 (i) (HSE 5.42$)$ \\
$2^{*}$ h-GaN & & & & & & \\
S-H & 3.45 & 0.29 & 2.01 & 1.61 & 1.04 & 6.32 & 3.44 (i) (HSE 4.73$)$ \\
DS-H & 3.24 & 0.72 & 2.01 & 1.56 & 1.03 & 6.86 & 3.19 (d) (HSE 4.44)
\end{tabular}
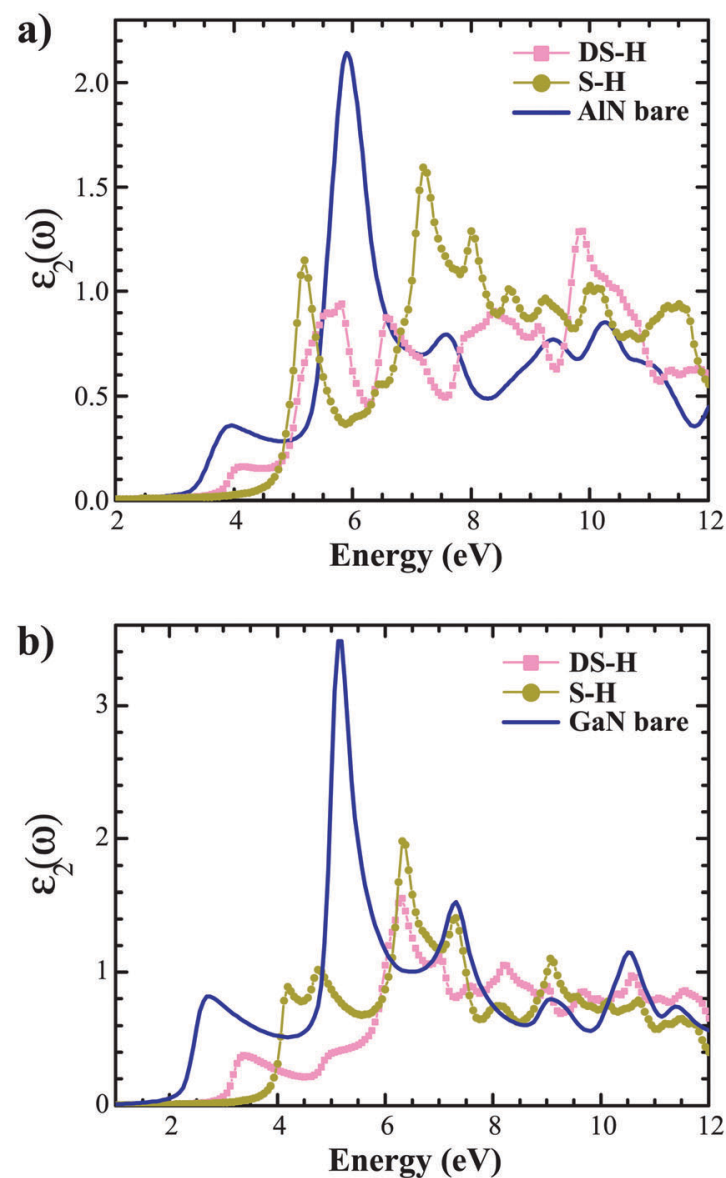

Fig. 10 (a) Imaginary dielectric function $\left[\varepsilon_{2}(\omega)\right]$ of bare, single-side (S-H) and double-side (DS-H) hydrogenated monolayer h-AIN; (b) $\varepsilon_{2}(\omega)$ of bare, $\mathrm{S}-\mathrm{H}$ and DS-H hydrogenated monolayer $\mathrm{h}-\mathrm{GaN}$.

mesh and coverage of $V_{\mathrm{F}}$ defects. Briefly, these three types of defects can lead to modifications in the band gap and hence attributes diverse functionalities to h-AlN and h-GaN.

\section{Hydrogenation of monolayers}

Full hydrogen coverage of h-AlN and h-GaN monolayers is of particular interest, since the physical and chemical properties of the bare material can be modified to a great extent. Owing to the regularities of the atomic structure, electronic band properties in $k$-space can be regained. Monolayers can retain their stability for specific hydrogenation geometries. Also the hydrogenation-dehydrogenation processes by themselves can be reversible and realized for many cycles. Thus, original and bare material properties can be regained by dehydrogenation following an annealing process. In the past, several $2 \mathrm{D}$ monolayer structures were seen to exhibit important changes in their electronic and magnetic properties upon hydrogenation. For instance, the planar structure of graphene becomes buckled and undergoes a metal-insulator transition. ${ }^{49}$ In contrast, buckled phosphorene and arsenene turn to 2D planar upon full hydrogenation and they attain a Dirac cone at the $K$ symmetry point..$^{50}$ This situation occurs also in fully hydrogenated antimonene and bismuthene. ${ }^{51}$

In the present case, the following five possible configurations of hydrogen coverage can occur on h-AlN and h-GaN: only $\mathrm{N}$ atoms are hydrogenated, which is specified as $(\mathrm{N}-\mathrm{H})$; only cation or metal atoms are hydrogenated $(\mathrm{Al} / \mathrm{Ga}-\mathrm{H})$; both cation and anion atoms at the same side of the monolayer are hydrogenated (single-sided, S-H); cations and anions are hydrogenated at opposite sides (double-sided, DS-H); two H atoms are adsorbed to each cation and anion from either side (F-H). All these configurations are examined by structure optimization and performing phonon calculations to test their dynamical stability. Among the five configurations, only the S-H and DS-H configurations are found to be stable; the other three were found to be unstable, since they had imaginary frequencies for specific phonon modes.

In Fig. 9, we present stable configurations together with their calculated phonons and electronic energy bands. Upon hydrogenation the planar geometry of h-AlN and h-GaN becomes buckled, since planar $\mathrm{sp}^{2}$ hybrid orbitals change to tetrahedrally coordinated $\mathrm{sp}^{3}$-like orbitals. The buckling height increases from $\sim 0.3 \AA$ to $\sim 0.7 \AA$ by going from S-H to DS-H, since the anion- $\mathrm{H}$ bond is stronger than the cation- $\mathrm{H}$ bond. In addition, the lattice constants of h-AlN and h-GaN differ in S-H and DS-H hydrogenation. As for the electronic structure, the band gaps of h-AlN and h-GaN increase upon hydrogenation. In Table 5 the values, such as structural parameters, cohesive energy and band gap calculated for S-H and DS-H hydrogenated monolayers are tabulated. In the same table, the fundamental band gaps calculated by HSE corrections over the PBE results are also given. Interestingly, differences between HSE and PBE band gaps of h-AlN and h-GaN covered by hydrogen in different decorations keep the values in the range of $\sim 1.2-1.3 \mathrm{eV}$ the 
same as for pristine h-AlN and h-GaN as seen in Section 3. This situation justifies the trend known as "scissor operation" that the difference between the HSE band gap (predicting a value relatively closer to the experimental value) and PBE band gap of defected systems comprising 50 atoms will be in the range of $\sim 1.2-1.3 \mathrm{eV}$. In ESI, $\dagger$ Fig. S2 the configuration of hydrogenation, which lead to dynamical instability and related phonon calculations are presented.

\subsection{Optical Properties of hydrogenated monolayers}

In order to compute the effects of hydrogenation on the optical properties, Random Phase Approximation (RPA) ${ }^{52,53}$ is employed on top of the PBE approach, using a sufficient total number of (valence and conduction) bands of 96. The linear optical response is calculated in terms of the isotropic complex dielectric function, composed of the real and imaginary parts, which can be derived from one another via Kramer-Kronigs relations. Monkhorst-Pack $k$-point sampling adopted for the PBE-RPA calculations was $127 \times 127$ in the $2 \mathrm{D}$ unitcell. Two different cases, namely S-H and DS-H were considered. The calculated, imaginary dielectric functions $\left[\varepsilon_{2}(\omega)\right]$ are shown in Fig. 10. Functionalizing monolayer h-AlN and h-GaN by full $\mathrm{H}$ coverage was observed to affect the in-plane optical response to a significant extent. As explained in detail in ref. 18, 54 and 55, critical features in the optical spectra of pristine monolayer h-AlN and h-GaN are especially the absorption onsets slightly above $3 \mathrm{eV}$ and around $2 \mathrm{eV}$, as well as the main optical absorption peaks appearing near $5.8 \mathrm{eV}$ and $5.2 \mathrm{eV}$, respectively. The absorption band edge of h-AlN was blue-shifted to $3.8 \mathrm{eV}$ for the DS-H case. On the other hand, the onset is further shifted above $4.6 \mathrm{eV}$ for the $\mathrm{S}-\mathrm{H}$ case. Moreover, the absorption peaks are scattered to multiple ones once h-AlN is hydrogenated, where the first main luminescence peaks appeared at $5.7 \mathrm{eV}$ and $5.2 \mathrm{eV}$, for the DS-H and S-H cases, respectively. Similarly, the band edge of bare h-GaN is blue-shifted to nearly $3.1 \mathrm{eV}$ and $3.9 \mathrm{eV}$ for DS-H and S-H absorbed h-GaN, respectively. Also, the main luminescence peak of bare GaN is degraded by amplitude, and blueshifted to around $6.4 \mathrm{eV}$ once functionalized by $\mathrm{H}$-coverage. Even though the positions of the onsets and luminescence peaks are not as exact as possible due to the gap underestimation of the PBE method, the results are still sufficient for a quantitative evaluation. Nevertheless, HSE corrections made over the PBE bands of pristine and hydrogenated h-AlN and h-GaN suggest that all adsorption edges of the curves in Fig. 10 should be blue-shifted by $1.2-1.3 \mathrm{eV}$.

In summary, the principal effect of hydrogenation of layered h-AlN and h-GaN structures has been to further shift the optical activity towards higher energies, allowing for optical
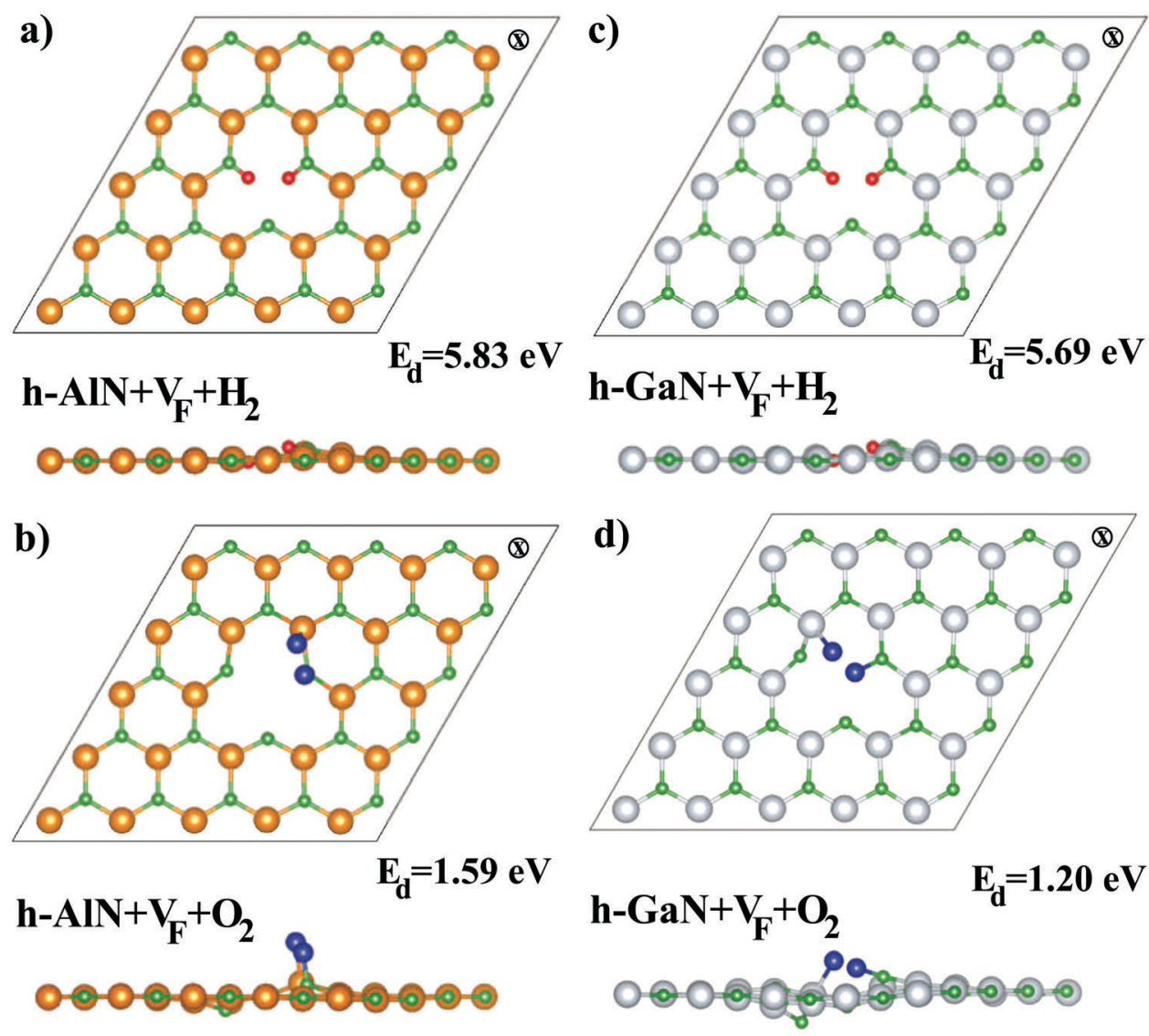

Fig. 11 (a) Top and side view of the atomic configurations after the spontaneous dissociations of a $\mathrm{H}_{2}$ molecule at the cation vacancy site of $\mathrm{h}$-AlN into constituent $\mathrm{H}$ atoms. The anion pair of the vacancy is indicated by " $\mathrm{x}$ ". The calculated dissociation energy $E_{\mathrm{d}}$, is also given. Hydrogen atoms are indicated by small red balls. (b) The same for the dissociation of $\mathrm{O}_{2}$ molecules at the cation vacancy site of h-AIN. Oxygen atoms are indicated by large blue balls. (c) The same for the dissociation of $\mathrm{H}_{2}$ at the cation vacancy site of $\mathrm{h}-\mathrm{GaN}$. (d) The same for the dissociation of $\mathrm{O}_{2}$ at the cation site of $\mathrm{h}-\mathrm{GaN}$. 
modulation by hydrogenation in the ultra-violet (UV) regime, indicating potential use in optoelectronic devices operating in the UV zone.

\section{Dissociation of $\mathrm{H}_{2}$ and $\mathrm{O}_{2}$}

Various orientations and geometries of $\mathrm{H}_{2}$ and $\mathrm{O}_{2}$ molecules approaching the cation vacancy site of the monolayers are examined. $\mathrm{A}_{2}$ molecule can be dissociated with a dissociation energy, $E_{\mathrm{d}}$ of $5.83 \mathrm{eV}$ at the $\mathrm{Al}$ vacancy site, when it is initially placed parallel to the h-AlN monolayer as shown in Fig. 11(a). Otherwise the $\mathrm{H}_{2}$ molecule weakly attaches to h-AlN over Al or $\mathrm{N}$ with about $0.10 \mathrm{eV}$ binding energy as summarized in ESI, $\uparrow$ Fig. S3. The $\mathrm{O}_{2}$ molecule also can dissociate both at the Al vacancy site when it is initially placed parallel to the monolayer near the top site of $\mathrm{N}$. After dissociation one $\mathrm{O}$ atom becomes bound to one $\mathrm{N}$ atom surrounding the vacancy, and the other $\mathrm{O}$ atom is attached to $\mathrm{Al}$ atom as shown in Fig. 11(b). The calculated dissociation energy is $1.59 \mathrm{eV}$, which is smaller than the binding energy of $\mathrm{O}_{2}$ due to the formation of $\mathrm{Al} / \mathrm{Ga}-\mathrm{O}$ and $\mathrm{N}-\mathrm{O}$ bonds. Different initial positions of the oxygen molecule relative to the vacancy site, which led to one case of dissociation with relatively smaller energy of dissociation and various cases of physisorption, are summarized in ESI, $\uparrow$ Fig. S4.

Interaction of $\mathrm{H}_{2}$ and $\mathrm{O}_{2}$ molecules with the h-GaN monolayer leading to either dissociation or physisorption is investigated in a similar manner. $\mathrm{H}_{2}$ can dissociate when it approaches the $\mathrm{Ga}$ vacancy site in a vertical or lateral orientation relative to the monolayer. Upon dissociation, constituent $\mathrm{H}$ atoms are bound to different $\mathrm{N}$ atoms surrounding the vacancy with $E_{\mathrm{d}}=5.69 \mathrm{eV}$. Other geometries of approach leading to dissociation or physisorption are presented in ESI, $\dagger$ Fig. S5. When the $\mathrm{O}_{2}$ molecule is initially placed parallel to the $\mathrm{h}$-GaN monolayer between two surrounding $\mathrm{N}$ atoms, it dissociates with $E_{\mathrm{d}}=1.20 \mathrm{eV}$; one of the constituent $\mathrm{O}$ atoms is bound to $\mathrm{Ga}$, the other one to the surrounding $\mathrm{N}$ atom. Other configurations leading to the bouncing of $\mathrm{O}_{2}$ from the monolayer or its physisorption are presented in ESI, $\dagger$ Fig. S6.

\section{Conclusions}

h-AlN and h-GaN monolayers are known to be indirect, wide band gap semiconductors with planar geometry. These monolayer materials can acquire diverse electronic, magnetic and optical properties upon chemical and substitutional doping, and formation of anti-site and vacancy defects. Selected adatoms $(\mathrm{H}, \mathrm{C}, \mathrm{N}, \mathrm{O}, \mathrm{F}, \mathrm{Al}, \mathrm{Si}, \mathrm{Ga}, \mathrm{Ge}$ and $\mathrm{As})$ readily form chemical bonds with these bare monolayers. The process of binding is exothermic with a positive binding energy ranging between $1.0 \mathrm{eV}$ and $4.0 \mathrm{eV}$. The contribution of the van der Waals interaction in these bonds is generally minute. As a result of chemical binding, local reconstructions can be induced or asymmetric dumbbell-like structures can be constructed at the close proximity of the defect. The stability of the dumbbell-like constructions are confirmed by finite temperature, $a b$ initio molecular dynamics calculations. The chemical doping of the GaN bilayer in the heackelite structure by specific adatoms breaks the vertical bonds and hence transforms it to a planar bilayer. The substitutional doping of atoms $(\mathrm{C}, \mathrm{N}, \mathrm{O}, \mathrm{Mg}, \mathrm{Al}, \mathrm{Si}$, $\mathrm{Ge}, \mathrm{Cr}, \mathrm{Ni}$ and $\mathrm{Zn}$ ) is generally endothermic, except for the $\mathrm{O}$ atom in h-AlN and $\mathrm{O}, \mathrm{Al}$ and $\mathrm{Si}$ atoms in h-GaN.

Since the coupling with nearest dopants or defects is negligible in the low (dilute) concentration limit, filled and/or empty localized, spin-polarized or spin-degenerate states can be induced in the band gap of the pristine monolayers. The resulting donor or acceptor states can modify the electronic and optical properties. In addition, the monolayers can attain integer numbers of local magnetic moment through broken spin-degeneracy. At a high concentration of dopant and defects, induced localized states broaden into spin-bands in the fundamental band gap. In particular, the bands originating from spin-polarized, localized states can attribute half-metallicity, when their coupling is significant. For example, fluorine adatoms adsorbed on h-AlN and h-GaN to form a periodic $(2 \times 2)$ mesh change these wide band gap semiconductors into half-metals. While spin-up bands form a semiconductor with band gaps wider than that of the pristine monolayers, spin-down bands are metallic. On the other hand, the pristine monolayers can change into either magnetic metals or magnetic narrow band gap semiconductors, when decorated by specific adatoms.

In addition to the doping, the formation of anti-sites and pairs of nearest and distant cation-anion vacancies can also lead to critical changes in the electronic structure by inducing localized gap states. While a cation-anion vacancy induces a permanent local magnetic moment of $2.0 \mu_{\mathrm{B}}$, an anti-site defect is nonmagnetic.

At the full coverage of $\mathrm{H}$ atoms, planar monolayers acquire buckling and remain stable only for specific geometries of coverage. For stable structures, the band gaps are generally widened with modified optical properties, but for a specific hydrogenation geometry the band gap changes from an indirect to direct one.

Interestingly, even if the binding of $\mathrm{H}_{2}$ and $\mathrm{O}_{2}$ molecules to the surface of bare monolayers is usually weak, these molecules can be dissociated spontaneously at the vacancy site; subsequently chemically active, unsaturated $\mathrm{sp}^{2}$ hybrid orbitals form strong bonds with constituent $\mathrm{H}$ and $\mathrm{O}$ atoms.

It should be noted that several earlier studies using the same theoretical method were demonstrated to be successful in predicting diverse properties, and several predictions in this work are relevant. Even though the underestimation of the band gap by DFT may make our results related to the electronic energy structure questionable, the trend that the difference between the real band gap and PBE band gap remains unchanged is crucial to interpret the results obtained within PBE. In particular, the position of the Fermi level relative to the conduction band edge of the pristine monolayer can be deduced from this trend.

In summary, our study demonstrates that monolayers of h-AlN and h-GaN in a honeycomb structure can acquire diverse changes and functionalities through doping, patterned adatom coverage and anti-site and vacancy defects, which can be exploited in 2D optoelectronics. We believe that the findings of this study will provide insight for the development of novel 2D materials. 


\section{Conflicts of interest}

There are no conflicts to declare.

\section{Acknowledgements}

The computational resources were provided by TUBITAK ULAKBIM, High Performance and Grid Computing Center (TR-Grid e-Infrastructure). S. C. acknowledges financial support from the Academy of Sciences of Turkey (TÜBA).

\section{References}

1 E. Durgun, S. Tongay and S. Ciraci, Phys. Rev. B: Condens. Matter Mater. Phys., 2005, 72, 075420.

2 S. Cahangirov, M. Topsakal, E. Akturk, H. Sahin and S. Ciraci, Phys. Rev. Lett., 2009, 102, 236804.

3 S. Cahangirov, M. Topsakal and S. Ciraci, Phys. Rev. B: Condens. Matter Mater. Phys., 2010, 81, 195120.

4 H. Sahin, S. Cahangirov, M. Topsakal, E. Bekaroglu, E. Akturk, R. T. Senger and S. Ciraci, Phys. Rev. B: Condens. Matter Mater. Phys., 2009, 80, 155453.

5 C. L. Freeman, F. Claeyssens, N. L. Allan and J. H. Harding, Phys. Rev. Lett., 2006, 96, 066102.

6 Y. Wang and S. Shi, Solid State Commun., 2010, 150, 1473-1478.

7 Y. Ma, Y. Dai, M. Guo, C. Niu, L. Yu and B. Huang, Appl. Surf. Sci., 2011, 257, 7845-7850.

8 A. K. Singh and R. G. Hennig, Appl. Phys. Lett., 2014, 105, 051604.

9 A. K. Singh, H. L. Zhuang and R. G. Hennig, Phys. Rev. B: Condens. Matter Mater. Phys., 2014, 89, 245431.

10 Q. Chen, H. Hu, X. Chen and J. Wang, Appl. Phys. Lett., 2011, 98, 053102.

11 Y. Gao, T. Yayama and S. Okada, Appl. Phys. Express, 2016, 9, 095201.

12 A. V. Kolobov, P. Fons, J. Tominaga, B. Hyot and B. Andre, Nano Lett., 2016, 16, 4849.

13 D. Wu, M. G. Lagally and F. Liu, Phys. Rev. Lett., 2011, 107, 236101.

14 H. L. Zhuang, A. K. Singh and R. G. Hennig, Phys. Rev. B: Condens. Matter Mater. Phys., 2013, 87, 165415.

15 D. V. Fakhrabad, N. Shahtahmasebi and M. Ashhadi, Superlattices Microstruct., 2015, 79, 38.

16 C. Bacaksiz, H. Sahin, H. D. Ozaydin, S. Horzum, R. T. Senger and F. M. Peeters, Phys. Rev. B: Condens. Matter Mater. Phys., 2015, 91, 085430.

17 D. Kecik, C. Bacaksiz, R. T. Senger and E. Durgun, Phys. Rev. B: Condens. Matter Mater. Phys., 2015, 92, 165408.

18 A. Onen, D. Kecik, E. Durgun and S. Ciraci, J. Phys. Chem. C, 2017, 121, 27098-27110.

19 A. Onen, D. Kecik, E. Durgun and S. Ciraci, Phys. Rev. B, 2017, 95, 155435.

20 D. Kecik, A. Onen, M. Konuk, E. Gurbuz, F. Ersan, S. Cahangirov, E. Akturk, E. Durgun and S. Ciraci, Appl. Phys. Rev., 2018, $5,01105$.
21 P. Tsipas, S. Kassavetis, D. Tsoutsou, E. Xenogiannopoulou, E. Golias, S. A. Giamini, C. Grazianetti, D. Chiappe, A. Molle, M. Fanciulli and A. Dimoulas, Appl. Phys. Lett., 2013, 103, 251605.

22 V. Mansurov, T. Malin, Y. Galitsyn and K. Zhuravlev, J. Cryst. Growth, 2015, 428, 93-97.

23 Z. Y. A. Balushi, K. Wang, R. K. Ghosh, R. A. Vila, S. M. Eichfeld, J. D. Caldwell, X. Qin, Y.-C. Lin, P. A. DeSario, G. Stone, S. Subramanian, D. F. Paul, R. M. Wallace, S. Datta, J. M. Redwing and J. A. Robinson, Nat. Mater., 2016, 15, 1166-1171.

24 K. T. Chan, J. B. Neaton and M. L. Cohen, Phys. Rev. B: Condens. Matter Mater. Phys., 2008, 77, 235430.

25 M. Sun, W. Tang, Q. Ren, S.-K. Wang, J. Yu and Y. Du, Appl. Surf. Sci., 2015, 356, 110-114.

26 T. T. Tran, K. Bray, M. J. Ford, M. Toth and I. Aharonovich, Nat. Nanotechnol., 2016, 11, 37.

27 F. Ersan, A. G. Gokce and E. Akturk, Appl. Surf. Sci., 2016, 389, 1. 28 F. Ersan, G. Gokoglu and E. Akturk, J. Phys.: Condens. Matter, 2014, 26, 325303.

29 C. Ataca and S. Ciraci, Phys. Rev. B: Condens. Matter Mater. Phys., 2012, 85, 195410.

30 D. C. Camacho-Mojica and F. Lopez-Urias, Chem. Phys. Lett., 2016, 652, 73.

31 W. Tang, M. Sun, J. Yu and J.-P. Chou, Appl. Surf. Sci., 2018, 427, 609-612.

32 F. Ersan, A. Akcay, G. Gokoglu and E. Akturk, Chem. Phys., 2015, 455, 73.

33 Y. Peng, C. Xia, H. Zhang, T. Wang, S. Wei and Y. Jia, J. Appl. Phys., 2014, 116, 044306.

34 C. Xia, Y. Peng, H. Zhang, T. Wang, S. Wei and Y. Jia, Acta Mater., 2013, 61, 7720-7725.

35 R. Gonzalez, W. L. Perez, A. Gonzalez-Garcia, M. G. MorenoArmenta and R. Gonzalez-Hernandez, Appl. Surf. Sci., 2018, 433, 1049-1055.

36 G. Kresse and J. Furthmüller, Phys. Rev. B: Condens. Matter Mater. Phys., 1996, 54, 11169.

37 G. Kresse and J. Furthmüller, Comput. Mater. Sci., 1996, 6, 15. 38 S. Grimme, J. Comput. Chem., 2006, 27, 1787-1799.

39 J. P. Perdew, K. Burke and M. Ernzerhof, Phys. Rev. Lett., 1996, 77, 3865.

40 P. E. Blöchl, Phys. Rev. B: Condens. Matter Mater. Phys., 1994, 50, 17953.

41 G. Kresse and D. Joubert, Phys. Rev. B: Condens. Matter Mater. Phys., 1999, 59, 1758.

42 H. J. Monkhorst and J. D. Pack, Phys. Rev. B: Condens. Matter Mater. Phys., 1976, 13, 5188.

43 J. Paier, M. Marsman, K. Hummer, G. Kresse, I. Gerber and J. Angyan, J. Chem. Phys., 2006, 124, 154709.

44 A. V. Krukau, O. A. Vydrov, A. F. Izmaylov and G. E. Scuseria, J. Chem. Phys., 2006, 125, 224106.

45 S. Cahangirov, V. O. Özçelik, A. Rubio and S. Ciraci, Phys. Rev. B: Condens. Matter Mater. Phys., 2014, 90, 085426.

46 V. O. Özçelik, E. Durgun and S. Ciraci, J. Phys. Chem. Lett., 2014, 5, 2694-2699.

47 Y. Yong, H. Cui, Q. Zhou, Y. K. X. Su and X. Li, RSC Adv., 2017, 7, 51027. 
48 H. Gao, H. Ye, Z. Yu, Y. Zhang and Y. Liu, Superlattices Microstruct., 2017, 112, 136.

49 D. C. Elias, R. R. Nair, T. M. G. Mohiuddin, S. V. Morozov, P. Blake, M. P. Halsall, A. C. Ferrari, D. W. Boukhvalov, M. I. Katsnelson, A. K. Geim and K. S. Novoselov, Science, 2009, 323, 610-613.

50 S. Zhang, Y. Hu, Z. Hu, B. Cai and H. Zeng, Appl. Phys. Lett., 2015, 107, 022102.
51 Z. Song, C.-C. Liu, J. Yang, J. Han, M. Ye, B. Fu, Y. Yang, Q. Niu, J. Lu and Y. Yao, NPG Asia Mater., 2014, 6, e147.

52 D. Bohm and D. Pines, Phys. Rev., 1951, 82, 625.

53 H. Ehrenreich and M. Cohen, Phys. Rev., 1959, 115, 786.

54 D. Kecik, C. Bacaksiz, R. T. Senger and E. Durgun, Phys. Rev. B: Condens. Matter Mater. Phys., 2015, 92, 165408.

55 A. Onen, D. Kecik, E. Durgun and S. Ciraci, Phys. Rev. B: Condens. Matter Mater. Phys., 2016, 93, 085431. 